ARTICLE

DOI: $10.1038 / \mathrm{s} 41467-017-02325-2$

\title{
Pro-inflammatory hepatic macrophages generate ROS through NADPH oxidase 2 via endocytosis of monomeric TLR4-MD2 complex
}

So Yeon Kim (1) ${ }^{1}$, Jong-Min Jeong ${ }^{1}$, Soo Jin Kim², Wonhyo Seo ${ }^{1}$, Myung-Ho Kim², Won-Mook Choi ${ }^{2}$, Wonbeak Yoo², Jun-Hee Lee ${ }^{2}$, Young-Ri Shim', Hyon-Seung $\mathrm{Yi}^{3}$, Young-Sun Lee (i) ${ }^{4}$, Hyuk Soo Eun ${ }^{3}$, Byung Seok Lee ${ }^{3}$, Kwangsik Chun ${ }^{5}$, Suk-Jo Kang ${ }^{6}$, Sun Chang Kim ${ }^{6,7}$, Bin Gao ${ }^{8}$, George Kunos ${ }^{8}, \mathrm{Ho} \mathrm{Min} \mathrm{Kim}^{2}$ \& Won-II Jeong 1,2

Reactive oxygen species (ROS) contribute to the development of non-alcoholic fatty liver disease. ROS generation by infiltrating macrophages involves multiple mechanisms, including Toll-like receptor 4 (TLR4)-mediated NADPH oxidase (NOX) activation. Here, we show that palmitate-stimulated $\mathrm{CD} 11 \mathrm{~b}^{+} \mathrm{F} 4 / 80^{\text {low }}$ hepatic infiltrating macrophages, but not $\mathrm{CD} 11 \mathrm{~b}^{+} \mathrm{F} 4$ / $80^{\text {high }}$ Kupffer cells, generate ROS via dynamin-mediated endocytosis of TLR4 and NOX2, independently from MyD88 and TRIF. We demonstrate that differently from LPS-mediated dimerization of the TLR4-MD2 complex, palmitate binds a monomeric TLR4-MD2 complex that triggers endocytosis, ROS generation and increases pro-interleukin-1 $\beta$ expression in macrophages. Palmitate-induced ROS generation in human CD68 ${ }^{\text {low } C D 14}$ high macrophages is strongly suppressed by inhibition of dynamin. Furthermore, Nox2-deficient mice are protected against high-fat diet-induced hepatic steatosis and insulin resistance. Therefore, endocytosis of TLR4 and NOX2 into macrophages might be a novel therapeutic target for non-alcoholic fatty liver disease.

\footnotetext{
${ }^{1}$ Laboratory of Liver Research, Biomedical Science and Engineering Interdisciplinary Program, KAIST, Daejeon 34141, Republic of Korea. ${ }^{2}$ Graduate School of Medical Science and Engineering, KAIST, Daejeon 34141, Republic of Korea. ${ }^{3}$ Department of Internal Medicine, Chungnam National University School of Medicine, Daejeon 35015, Republic of Korea. ${ }^{4}$ Department of Internal Medicine, Korea University College of Medicine, Seoul 08308, Republic of Korea. ${ }^{5}$ Department of Surgery, Chungnam National University School of Medicine, Daejeon 35015, Republic of Korea. ${ }^{6}$ Department of Biological Sciences, Korea Advanced Institute of Science and Technology, Daejeon 34141, Republic of Korea. ${ }^{7}$ Intelligent Synthetic Biology Center, 373-1, Guseong-dong, Yuseong-gu, Daejeon 34141, Republic of Korea. ${ }^{8}$ National Institutes of Health (NIH), National Institute on Alcohol Abuse and Alcoholism (NIAAA), Bethesda, MD 20892, USA. Correspondence and requests for materials should be addressed to W.-I.J. (email: wijeong@kaist.ac.kr)
} 
D iet-induced obesity is commonly associated with nonalcoholic fatty liver disease (NAFLD) and insulin resistance, in which recruited immune cells such as macrophages, neutrophils, lymphocytes, mast cells and eosinophils contribute to a pro-inflammatory environment ${ }^{1,2}$. Of these cells, recruited macrophages and resident Kupffer cells secrete diverse cytokines including tumor necrosis factor- $\alpha$ (TNF- $\alpha$ ), interleukin-6 (IL-6) and IL-1 $\beta$, which contribute to insulin resistance in hepatocytes by upregulating the activation of inhibitor of $\kappa \mathrm{B}$ kinase- $\beta /$ nuclear factor- $\kappa \mathrm{B}(\mathrm{NF}-\kappa \mathrm{B})$ and $\mathrm{c}$-Jun $\mathrm{N}$ terminal kinase $(\mathrm{JNK}) /$ activator protein $-1^{2-4}$. In this process, increased plasma levels of saturated free fatty acids (FFAs), such as palmitic acids, triggers pro-inflammatory signals through Tolllike receptor 2 (TLR2) and TLR4 in macropahges ${ }^{5}$.

Reactive oxygen species (ROS) are one of the major causes in steatohepatitis and insulin resistance. Infiltrated macrophages generate ROS by multiple mechanisms, including mitochondria damage, endoplasmic reticulum stress, and nicotinamide adenine dinucleotide phosphate (NADPH) oxidases (NOXs) ${ }^{6}$, 7. Among members of the NOX family, NOX2, also known as gp91phox, is the phagocytic NADPH oxidase, constitutively associated with p22phox in plasma membrane. Upon activation of NOX2, phosphorylated p47phox is translocated to p22phox, recruiting the additional components p67phox, p40phox and Rac GTPases to assemble a complex with NOX2, which then generates superoxide ${ }^{8}$. In liver resident Kupffer cells and infiltrating macrophages, NOX2derived ROS stimulates them to produce pro-inflammatory cytokines such as TNF- $\alpha$, IL- 6 , and IL- $1 \beta$, in response to various factors including oxidized low-density lipoprotein (LDL) and lipopolysaccharide (LPS $)^{9-11}$. In contrast to classically activated inflammatory macrophages, termed as M1, alternatively activated macrophages (M2) by Th2 cytokines IL- 4 and IL-13 secrete antiinflammatory cytokine IL-10, which protects from inflammationmediated insulin resistance by inhibiting the deleterious effects of pro-inflammatory cytokines on insulin signaling ${ }^{3}$. A phenotype switch from M1 to M2, associated with reduced ROS formation, is brought about by the absence NOX2 due to its diminished mRNA stability or genetic ablation ${ }^{11,12}$. Until now, the exact roles of NOX2 in inducing steatosis and insulin resistance in hepatic macrophages have not been clearly understood.

TLR4 plays a key role for innate immune responses and its signaling is triggered by the transfer of its ligand LPS to a TLR4-MD2 complex, which then undergoes homodimerization $^{13}$. This homodimer then can activate MyD88-dependent or MyD88-independent pathways. The MyD88-dependent pathway is activated at the plasma membrane, whereas the TRIFdependent (MyD88-independent) pathway requires internalization of TLR4 into endosome in a dynamin-dependent manner ${ }^{14}$, 15. In addition to LPS, TLR4 can bind a wide range of ligands including FFAs $2,3,5$. The mechanisms underlying TLR4 binding to diverse agonist are not clear, although the heterodimerization of TLR4 or the involvements of co-receptors and accessory proteins such as CD14, CD36 and MD2 have been suggested for the recognition of diverse TLR4 agonists ${ }^{14}$. Nonetheless, LPSmediated or FFA-mediated TLR4 signaling activates the same downstream transcriptional factors such as NF- $\kappa \mathrm{B}$ and interferon-regulatory factors to induce inflammatory responses ${ }^{14}$. Recently, a line of studies has suggested that sensing of LPS by TLR4 mediates priming and activation of several NOX family members, leading to enhanced inflammatory responses including ROS generation, the NLR family pyrin domain containing 3 (NLRP3) inflammasome-associated IL-1 $\beta$ production, and NF$\kappa \mathrm{B}-$ mediated IL- 6 production in mouse macrophages and human blood monocytes ${ }^{16-18}$. While the obesity-related increase in circulating FFA promotes the development of hepatic steatosis and insulin resistance, the precise molecular mechanisms involved in the interplay among FFA, TLR4 and NOX2 have not been fully understood.

Here, we report that in $\mathrm{CD} 11 \mathrm{~b}^{+} \mathrm{F} 4 / 80^{\text {low }}$ hepatic macrophages, palmitate triggers the endocytosis of a monomeric TLR4-MD2 complex, leading to NOX2 activation and ROS generation, which play an obligatory role in diet-induced hepatic steatosis and insulin resistance. These findings provide novel pathophysiological insights and therapeutic targets for NAFLD.

\section{Results}

NOX2 deficiency attenuates HFD-induced hepatic steatosis. To investigate the role of NOX2 in vivo, wild type (WT) and Nox2 KO mice were fed a high-fat diet (HFD) for 12 weeks. At sacrifice, body, liver and epididymal fat pad weights were significantly lower in Nox2 KO than WT mice, whereas food intake was similar in the two strains (Fig. 1a, b). On gross observation, livers of Nox2 KO mice were less yellowish in color than those of WT mice (Fig. 1b). Serum levels of alanine aminotransferase (ALT), aspartate aminotransferase (AST), triglyceride (TG) and total cholesterol (TC) were significantly lower in Nox2 KO than WT mice (Fig. 1c). Nox2 KO mice had less liver fat, as reflected in hepatic TG levels, and higher liver glycogen content than WT mice (Fig. 1d, e). Furthermore, protein levels of sterol regulatory element-binding protein-1 (SREBP1) and fatty acid synthase (FAS) were remarkably reduced, whereas levels of phosphorylated AMP kinase (AMPK) and AKT were significantly increased in Nox2 KO compared to WT mice, indicating increased insulin sensitivity in the former (Fig. 1f). Consistently, basal blood glucose was lower, and glucose tolerance and insulin sensitivity were higher in Nox2 KO than in WT mice, as quantified by glucose tolerance test (GTT) and insulin tolerance test (ITT), respectively (Fig. 1g). These differences were evident as early as 6 weeks of HFD feeding (Supplementary Fig. 1). These data suggest that NOX2 might be correlated with hepatic steatosis and insulin resistance in HFD-fed mice.

NOX2 deficiency decreases ROS production in $\mathrm{CD}_{11 b^{+} \mathrm{F} 4 /}$ $\mathbf{8 0}^{\text {low }}$ cells. Next, FACS analyses revealed that hepatic populations of lymphocytes such as NK cells $\left(\mathrm{NK} 1.1^{+} \mathrm{CD}^{-}\right)$, NKT cells $\left(\mathrm{NK} 1.1^{+} \mathrm{CD}^{+}\right), \mathrm{CD} 4 \mathrm{~T}$ cells $\left(\mathrm{CD}^{+} \mathrm{CD} 3^{+}\right), \mathrm{CD} 8 \mathrm{~T}$ cells $(\mathrm{CD} 8$ ${ }^{+} \mathrm{CD}^{+}$), and regulatory $\mathrm{T}$ cells (Treg; $\mathrm{CD} 4^{+} \mathrm{CD} 25^{+} \mathrm{Foxp}^{+}$) were similar between WT and Nox2 KO mice (Fig. 2a). However, the population of infiltrated $\mathrm{CD} 11 \mathrm{~b}^{+} \mathrm{F} 4 / 80^{\text {low }}$ macrophages was significantly decreased in the absence of NOX2, while $\mathrm{Ly}_{6 \mathrm{G}}{ }^{+} \mathrm{CD} 11 \mathrm{~b}$ + neutrophils and $\mathrm{CD} 11 \mathrm{~b}^{+} \mathrm{F} 4 / 80^{\text {high }}$ Kupffer cells showed no significant differences in livers of Nox2 $\mathrm{KO}$ and WT mice (Fig. 2b). ROS generation was remarkably decreased in CD11b ${ }^{+} \mathrm{F} 4 / 80^{\text {low }}$ macrophages of Nox2 KO compared to WT mice, whereas $\mathrm{CD}_{11 \mathrm{~b}} \mathrm{~F}^{+} \mathrm{F} / 80^{\text {high }}$ Kupffer cells produced similar level of ROS (Fig. 2c), suggesting the dominant role of $\mathrm{CD}_{11} \mathrm{~b}^{+} \mathrm{F} 4 / 80^{\text {low }}$ macrophages in ROS generation in response to HFD feeding. In parallel with these findings, quantitative RT-PCR (qRT-PCR) analyses showed that the expression of $T n f-\alpha$ and $I l-1 \beta$ was significantly lower in liver mononuclear cells (MNCs) of Nox2 KO mice compared to WT mice (Fig. 2d). Moreover, immunoblots of liver tissues revealed that phosphorylated NF- $\mathrm{KB}$ and JNK proteins were significantly reduced (Fig. 2e), which was paralleled by a significant reduction in apoptotic cells in Nox2 KO compared to $W T$ (Fig. 2f). However, in vitro exposure to palmitate did not induce apoptosis of $\mathrm{CD} 11 \mathrm{~b}^{+} \mathrm{F} 4 / 80^{\mathrm{low}}$ macrophages from either WT or Nox2 KO mice (Fig. $2 \mathrm{~g}$ ). Collectively, these data suggest that instead of resident $\mathrm{CD} 11 \mathrm{~b}^{+} \mathrm{F} 4 / 80^{\text {high }}$ Kupffer cells, CD11b ${ }^{+} \mathrm{F} 4 / 80^{\text {low }}$ macrophages are responsible for NOX2-mediated ROS generation in the liver of HFD-fed mice. 
a

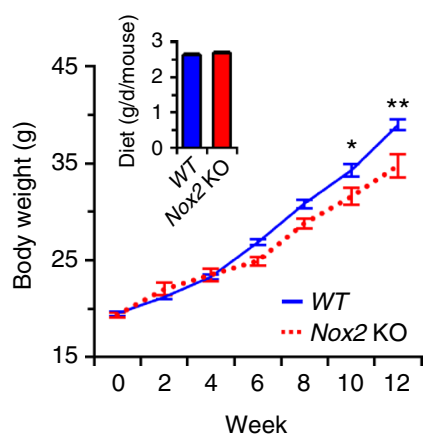

C

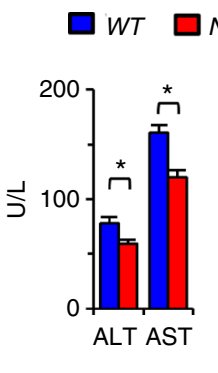

d
HFD (12 weeks)

Oil-red O

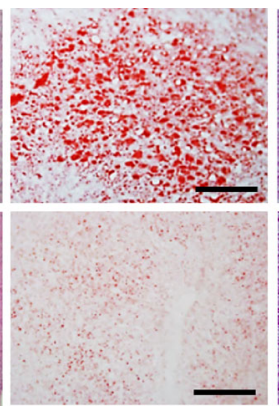

PAS

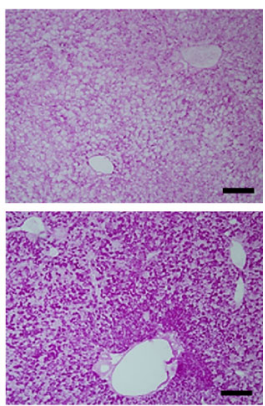

b
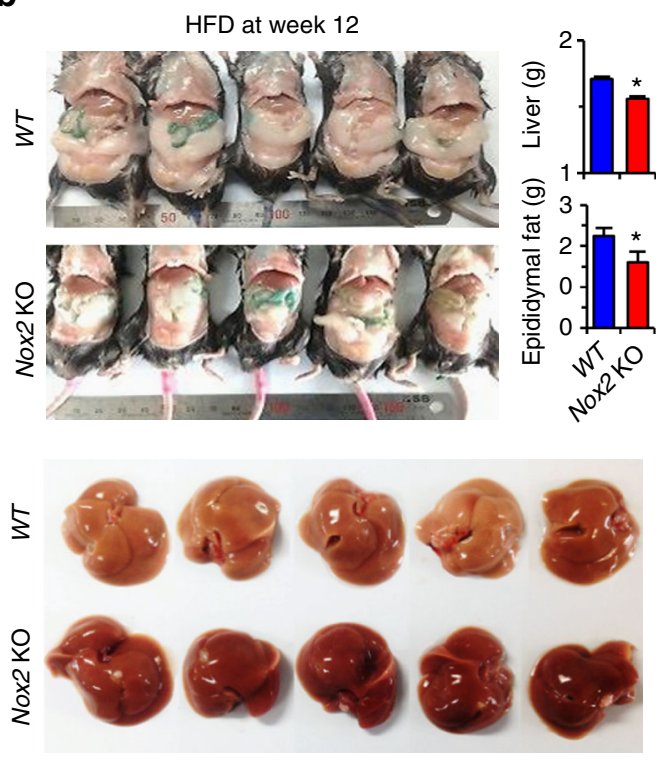

e

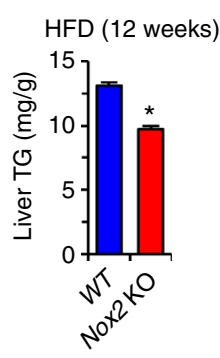

g

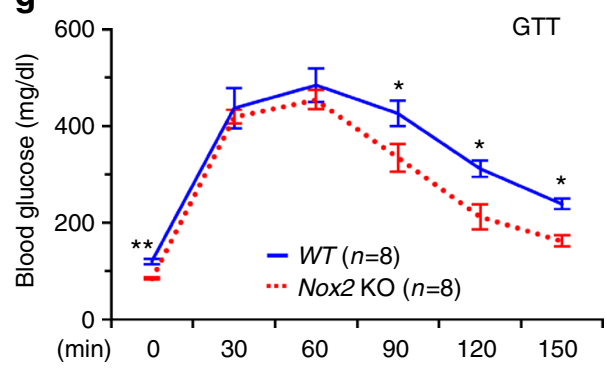

f

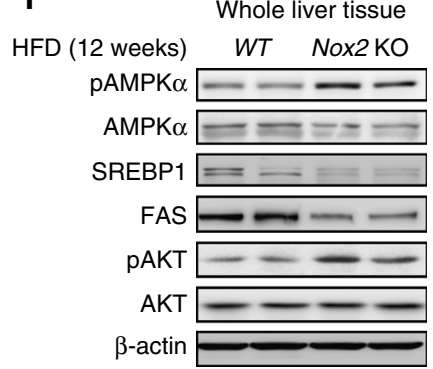

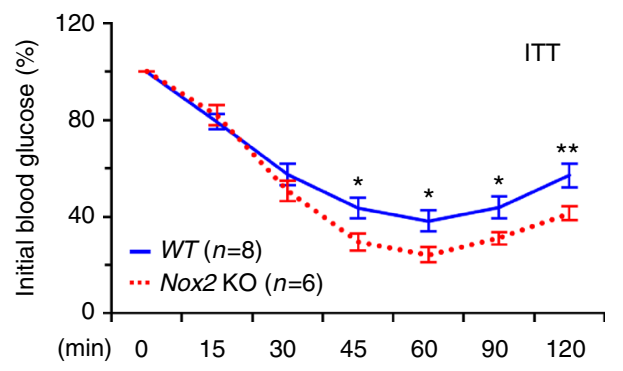

Fig. 1 Ablation of NOX2 ameliorates high-fat diet-induced hepatic steatosis in mice.WT and Nox2 KO mice were fed a high-fat diet for 12 weeks. a Changes in body weight and diet intake. b Representative gross findings and their weights of epididymal fat and liver in WT and Nox2 KO mice at week 12 . c Blood chemistry analyses for alanine aminotransferase (ALT), aspartate aminotransferase (AST), triglyceride (TG), and total cholesterol (TC). d Sectioned liver tissues stained with hematoxylin and eosin (H\&E), oil-red O (Oil-Red O), and periodic acid-Schiff (PAS). Bar = $100 \mu \mathrm{m}$. e TG levels measured in whole liver tissues. $\mathbf{f}$ Liver tissues subjected to Western blotting. $\mathbf{g}$ Glucose tolerance tests (GTTs) and insulin tolerance tests (ITTs) performed after $16 \mathrm{~h}$ of fasting.

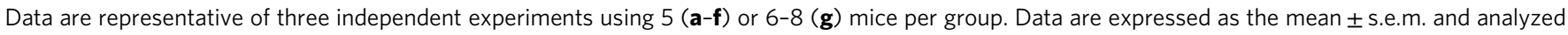
by Student's $t$-test, ${ }^{\star} P<0.05,{ }^{\star \star} P<0.01$ in comparison with the corresponding controls

Pro-inflammatory phenotype of $\mathrm{CD} 1 \mathrm{~b}^{+} \mathrm{F} 4 / 80^{\text {low }}$ macrophages. To validate the notion that $\mathrm{CD} 11 \mathrm{~b}^{+} \mathrm{F} 4 / 80^{\text {low }}$ macrophages rather than $\mathrm{CD} 11 \mathrm{~b}^{+} \mathrm{F} 4 / 80^{\text {high }}$ Kupffer cells are responsible for NOX2-dependent ROS generation in response to HFDmediated liver injury, macrophage polarization markers were analyzed in hepatic macrophages isolated from WT mice (C57BL/ 6 ), using fluorescence-activated cell sorting (FACS). As previously reported $^{19}$, two types of hepatic macrophages were identified as $\mathrm{CD} 11 \mathrm{~b}^{+} \mathrm{F} 4 / 80^{\text {high }}$ (or CD $11 \mathrm{~b}^{+} \mathrm{Ly}_{6 \mathrm{G}^{+}} \mathrm{Ly}_{6 \mathrm{C}^{\mathrm{low}}}$ ) Kupffer cells and $\mathrm{CD} 11 \mathrm{~b}^{+} \mathrm{F} 4 / 80^{\text {low }}$ (or CD11b ${ }^{+} \mathrm{Ly}_{6 \mathrm{G}^{-}} \mathrm{Ly}_{6 \mathrm{C}^{\text {high }}}$ ) macrophages (Fig. 3a). CD11b $b^{+} 4 / 80^{\text {high }}$ Kupffer cells showed higher expression of CD206 than $\mathrm{CD} 1 \mathrm{bb}^{+} \mathrm{F} 4 / 80^{\mathrm{low}}$ macrophages, with no expression detectable in hepatic lymphocytes, as a reflection of a more pro-inflammatory phenotype in infiltrating macrophages than in Kupffer cells (Fig. 3a). Next, we assessed morphological differences between Kupffer cells and macrophages by sorting and staining with Giemsa solution. Under the microscope, CD11b ${ }^{+} \mathrm{F} 4 / 80^{\mathrm{low}}$ macrophages were identified with their small cytoplasm and indented nuclei, whereas $\mathrm{CD} 11 \mathrm{~b}^{+} \mathrm{F} 4 / 80^{\text {high }}$ Kupffer cells showed abundant cytoplasm and marginal location of ovoid nuclei (Fig. 3b; Supplementary Fig. 2a, b). In qRT-PCR analyses, $\mathrm{CD} 11 \mathrm{~b}^{+} \mathrm{F} 4 / 80^{\text {low }}$ macrophages displayed higher expression of Nox2, Tlr4 and Cd14 than CD11b ${ }^{+}$F $4 / 80^{\text {high }}$ Kupffer cells (Fig. 3c). In addition, gene expression of pro-inflammatory markers such as $T n f-\alpha$ and $I l-1 \beta$ was higher, whereas expression of $\mathrm{Il}-10$ was significantly lower in $\mathrm{CD} 11 \mathrm{~b}^{+} \mathrm{F} 4 / 80^{\text {low }}$ macrophages than in $\mathrm{CD}_{11} \mathrm{~b}^{+} \mathrm{F} 4 / 80^{\text {high }}$ Kupffer cells (Fig. 3d). Interestingly, similar findings were still detected in freshly isolated hepatic macrophages and Kupffer cells isolated from mice fed a HFD for 12 weeks (Supplementary Fig. 2c). Histogram data showed that expression of TLR4 was similar but expression of CD14 and CD11c was higher in macrophages than those of Kupffer cells (Fig. 3e). All these data indicate that $\mathrm{CD} 11 \mathrm{~b}^{+} \mathrm{F} 4 / 80^{\mathrm{low}}$ macrophages show more inflammatory phenotype than $\mathrm{CD} 11 \mathrm{~b}^{+} \mathrm{F} 4 /$ $80^{\text {high }}$ Kupffer cells in liver of HFD-fed WT mice.

Endocytosis of TLR4 and NOX2 in $\mathrm{CD} 11 \mathrm{~b}^{+} \mathrm{F} 4 / 80^{\text {low }}$ cells by palmitate. To explore the roles of TLR4 and NOX2 in ROS generation, isolated macrophages and Kupffer cells of WT, Tlr4 KO and Nox2 KO mice were treated with palmitate and then ROS generation 
a

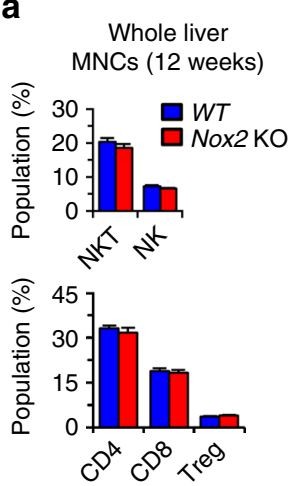

d Liver MNCs (12 weeks)

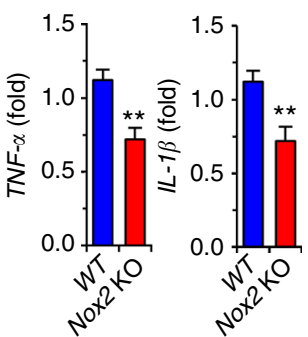

b

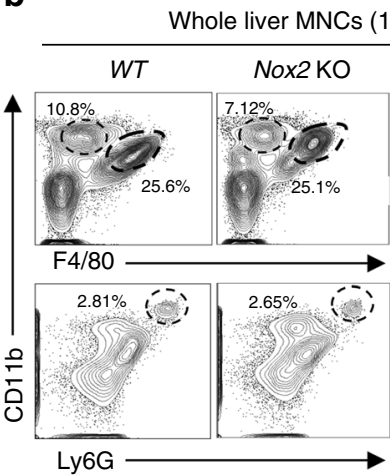

e

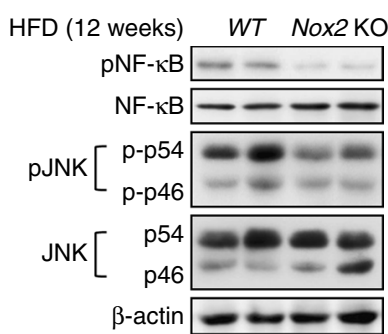

C

Freshly isolated cells (12 weeks)

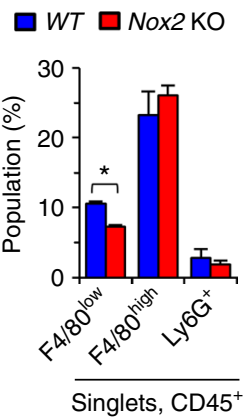

$\mathbf{f}$

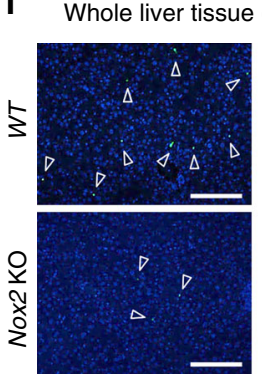

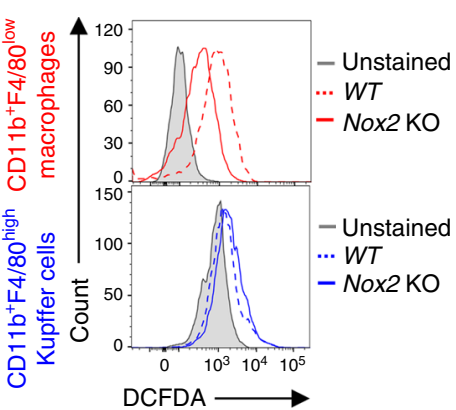

DCFDA

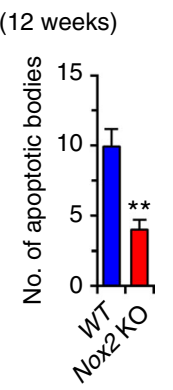
CD $11 b^{+} F 4 / 80^{\text {low }}$ cells $(1 \mathrm{~h})$

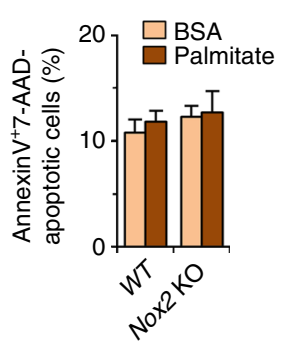

Fig. 2 NOX2 deficiency decreases inflammatory response in CD11b ${ }^{+}$44/80 low macrophages in mice fed a high-fat diet.WT and Nox2 KO mice were fed a high-fat diet for 12 weeks. a, b Isolated whole liver MNCs were subjected to flow cytometry analyses. c The generation of ROS was monitored by DCF fluorescence in freshly isolated CD11 $\mathrm{b}^{+} \mathrm{F} 4 / 80^{\text {high }}$ Kupffer cells and $C D 11 \mathrm{~b}^{+} \mathrm{F} 4 / 80^{\text {low }}$ macrophages. d, e Whole liver MNCs and tissues were subjected to qRT-PCR and Western blotting, respectively. $\mathbf{f}$ Apoptotic bodies were assessed and counted after TUNEL staining (average number of 5 fields under $\times 200$ magnification). Bar $=200 \mu \mathrm{m}$. g After treatment with palmitate for $1 \mathrm{~h}, \mathrm{CD} 11 \mathrm{~b}^{+} \mathrm{F} 4 / 80^{\mathrm{low}}$ macrophages stained with Annexin $\mathrm{V}$ and $7-A A D$ were analyzed by flow cytometry. Data are representative of three independent experiments using $5(\mathbf{a}-\mathbf{f})$ and 3 (g) mice per group. Data are expressed as the mean $\pm \mathrm{s}$. e.m. and analyzed by Student's $t$-test, ${ }^{\star \star} P<0.01$ in comparison with the corresponding controls

was assessed. As expected, $\mathrm{CD} 11 \mathrm{~b}^{+} \mathrm{F} 4 / 80^{\text {low }}$ macrophages of $W T$ mice produced ROS, while $\mathrm{CD} 11 \mathrm{~b}^{+} \mathrm{F} 4 / 80^{\text {low }}$ macrophages of Nox2 $\mathrm{KO}$ and Tlr4 KO mice did not (Fig. 4a; Supplementary Fig. 3a). More interestingly, $\mathrm{CD} 11 \mathrm{~b}^{+} \mathrm{F} 4 / 80^{\text {high }}$ Kupffer cells from any of the three strains (WT, Tlr4 KO and Nox2 KO mice) failed to produce ROS in response to palmitate treatment (Fig. 4a). In qRT-PCR analyses, dynamin expression was significantly increased in $\mathrm{CD} 11 \mathrm{~b}$ ${ }^{+} \mathrm{F} 4 / 80^{\text {low }}$ macrophages compared with $\mathrm{CD} 11 \mathrm{~b}^{+} \mathrm{F} 4 / 80^{\text {high }}$ Kupffer cells in WT mice (Fig. 4b). Blocking dynamin by dynasore resulted in a significant reduction of $\mathrm{ROS}$ generation in $\mathrm{CD} 11 \mathrm{~b}^{+} \mathrm{F} 4 / 80^{\text {low }}$ macrophages of $W T$ mice (Fig. 4c).

Next, we analyzed RAW 264.7 mouse macrophages because of their similarity to $\mathrm{CD} 11 \mathrm{~b}^{+} \mathrm{F} 4 / 80^{\text {low }}$ macrophages (Fig. $4 \mathrm{~d}$ ). Palmitate treatment of RAW 264.7 cells increased ROS generation and Il-1 $1 \beta$ expression and these effects were abrogated by dynasore treatment (Fig. 4e). Immunostaining revealed the wide distribution but discrete localization of TLR4 and NOX2 proteins in the cytoplasm of control RAW 264.7 macrophages (Fig. 4f, upper lane). Palmitate treatment resulted in the internalization of these proteins to nucleus and also their increased co-localization in the perinuclear areas (Fig. 4f, middle lane). Dynasore treatment inhibited the internalization and co-localization of these proteins (Fig. 4f, lower lane; Supplementary Fig. 3b). These data suggest that dynamin-dependent endocytosis of both NOX2 and TLR4 proteins is important for ROS generation in macrophages, but not Kupffer cells, in response to palmitate treatment.

Palmitate-mediated ROS is produced in a TLR4/NOX2dependent manner. Immunoblots of RAW 264.7 cells revealed palmitate-induced significant internalization of TLR4 and NOX2 proteins from membrane fraction to cytosol, but a direct interaction between TLR4 and NOX2 proteins was not observed (Fig. 5a). In addition, phosphorylation of NF-KB and JNK (pNF$\kappa \mathrm{B}$ and $\mathrm{pJNK}$ ) and expression of Nlrp3 and $\mathrm{Il}-1 \beta$ mRNAs were increased by palmitate treatment, but these effects were absent in Nox2- or Tlr4-depleted RAW 264.7 cells (Fig. 5a; Supplementary Fig. 4a-d). In parallel, palmitate treatment induced not only caspase-1 activation but also increased expression of $\mathrm{Il}-1 \beta$ and Nlrp3 in WT bone marrow-derived macrophages (BMDMs), but not in Nox2 KO BMDMs (Fig. 5b, c). Next, we investigated molecular mechanisms of TLR4/NOX2-mediated ROS generation. In response to palmitate, siRNA-mediated depletion of Tlr4, $M d 2$, Nox2, Rac1, and Rac2 abolished ROS production, whereas treatment with siRNA against Cd14, Myd88, and Trif or the inhibition of Cd36, fatty acid translocase, did not affect ROS production (Fig. 5d; Supplementary Fig. 4e).

To further test whether palmitate induces MyD88/TRIFindependent, TLR4/NOX2-dependent ROS generation in vivo, we perfused the liver of WT, Tlr4 KO, Nox2 KO and Myd88/Trif double $\mathrm{KO}$ mice via the portal vein with BODIPY-labeled fatty acid analogs (C16-BODIPY) or palmitate. During the last minute of perfusion, superior and inferior vena cava were clipped so that the liver remained exposed to the perfusate, followed by rapid isolation of hepatic immune cells (Fig. 5e). We verified that C16BODIPY was successfully delivered to all hepatic immune cells with high Nox2 expression, including macrophages, Kupffer cells and neutrophils (Fig. 5f, lower panels; Supplementary Fig. 4f). However, only CD11b ${ }^{+} \mathrm{F} 4 / 80^{\text {low }}$ macrophages generated ROS in response to palmitate infusion (Fig. 5f, upper panels). 
a

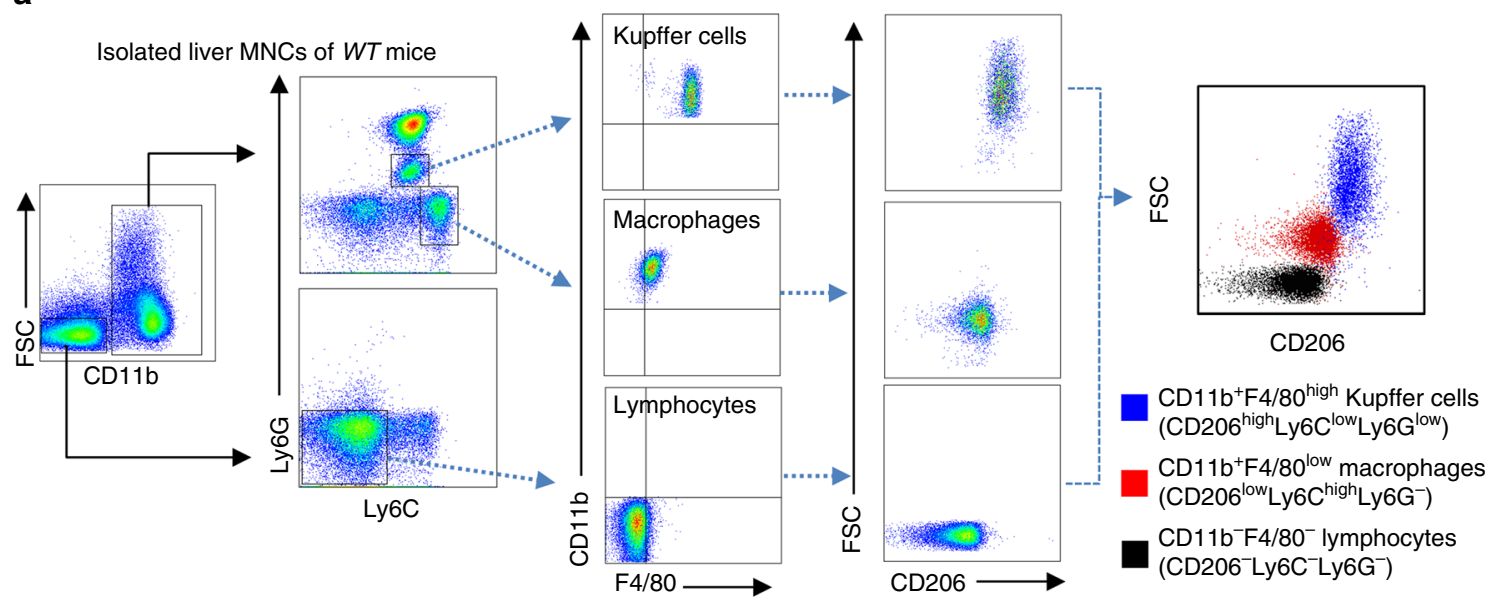

b

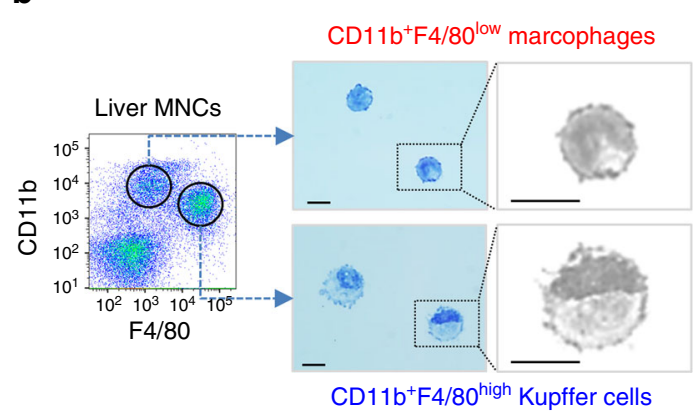

C

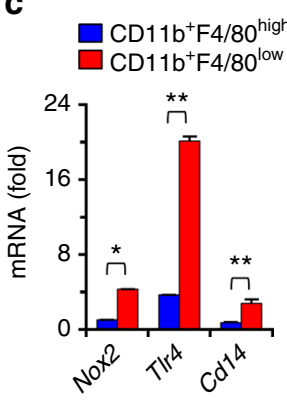

d

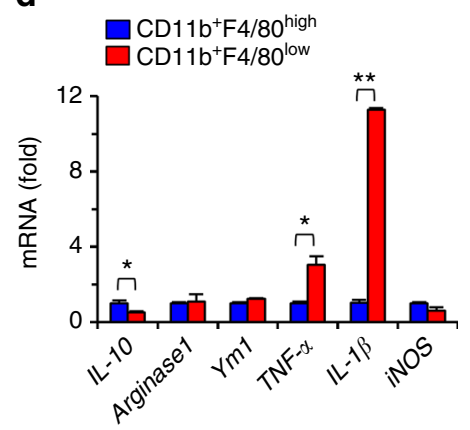

e

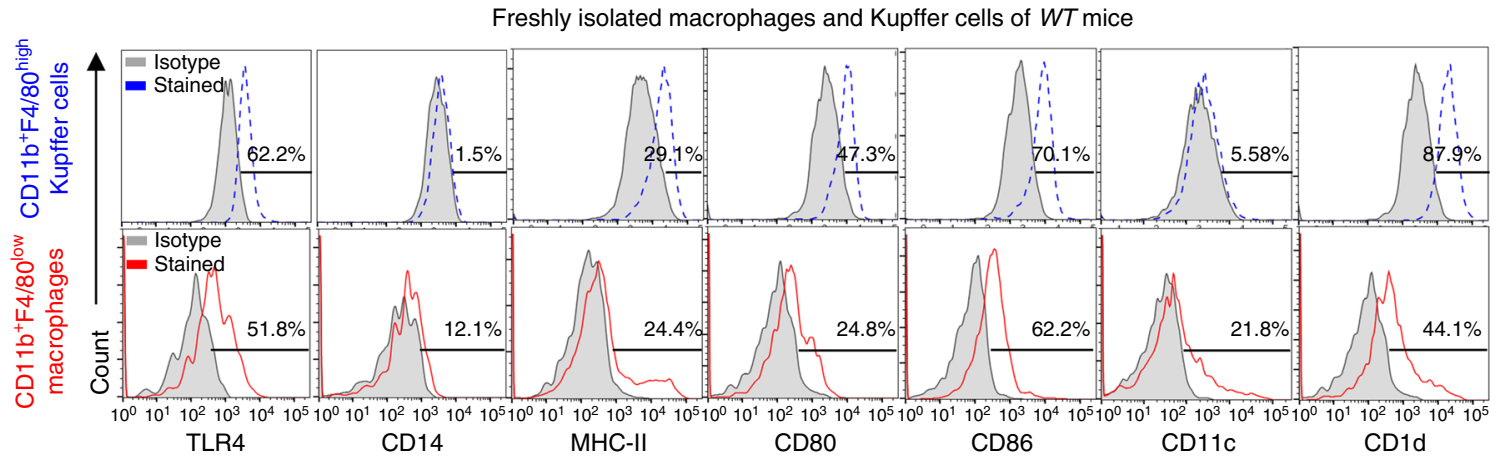

Fig. $3 \mathrm{CD} 11 \mathrm{~b}^{+} \mathrm{F} 4 / 80^{\text {low }}$ macrophages present a more pro-inflammatory phenotype than $\mathrm{CD} 11 \mathrm{~b}^{+} \mathrm{F} 4 / 80^{\text {high }}$ Kupffer cells.a Flow cytometry analysis of freshly isolated liver mononuclear cells (MNCs) stained for monocyte lineage markers Ly6C, F4/80, CD11b and CD206 from control WT mice. Prior to CD11b ${ }^{+}$ gating, singlet and live cell gating were performed. b Isolated cells were visualized by Giemsa staining. Bar $=10 \mu \mathrm{m}$. $\mathbf{c}$ The gene expression of Nox2, T/r 4 and Cd14 in Kupffer cells and macrophages. d Relative expression of pro-inflammatory genes in Kupffer cells and macrophages. e Flow cytometry analysis of

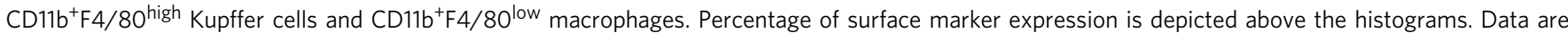
representative of three independent experiments in vitro using isolated liver immune cells from 3 mice per group. Data are expressed as the mean \pm s.e.m. and analyzed by Student's $t$-test, ${ }^{\star} P<0.05,{ }^{\star}{ }^{\star} P<0.01$ in comparison with the corresponding controls

Interestingly, $\mathrm{CD}_{11} \mathrm{~b}^{+} \mathrm{F} 4 / 80^{\text {low }}$ macrophages from $W T$ and Myd88/Trif double KO mice produced similar levels of ROS in response to palmitate, whereas no such effect was observed in Tlr4 KO or Nox2 KO mice (Fig. 5g). All this suggests that palmitate treatment generates ROS by a MyD88/TRIFindependent, TLR4/NOX2-dependent manner.

Palmitate binding to the monomeric TLR4-MD2 complex in macrophages. To investigate direct binding of palmitate to the TLR4-MD2 complex, purified recombinant TLR4-MD2, $\mathrm{MD} 2$, and CD14 from Hi5 insect cells were incubated with C16-BODIPY as previously reported ${ }^{20,} 21$ and were subjected to native polyacrylamide gradient gel electrophoresis (Native PAGE). Strong fluorescence signal was observed in C16-BODIPY plus TLR4-MD2 complex, which was co-localized with the position of TLR4-MD2 complex as visualized by Commassie stain, indicating that C16-BODIPY can bind to TLR4-MD2 but not to CD14 and MD2 (Supplementary Fig. 5).

Based on the above findings, we further investigated whether palmitate binding can induce the dimerization of TLR4-MD2 complex, similar to LPS. In size-exclusion chromatography, a representative peak of monomeric human TLR4-MD2 
a

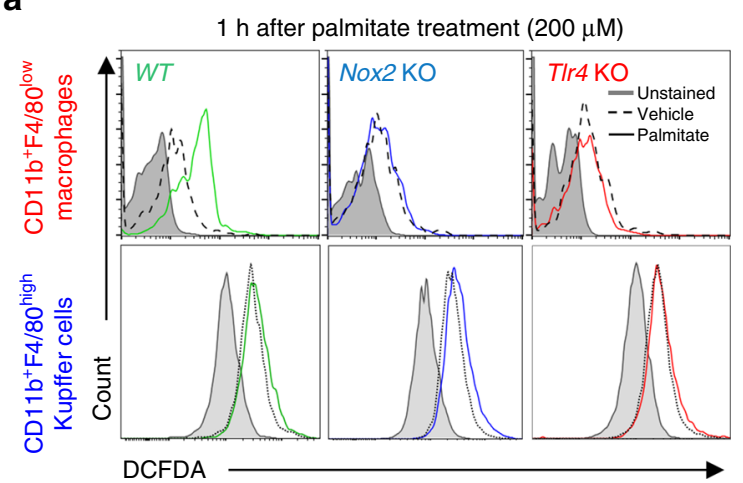

b

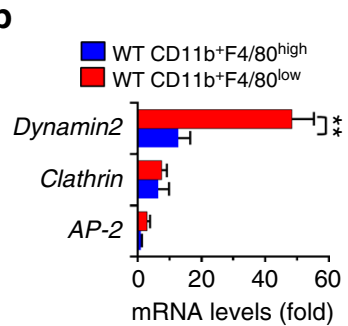

C

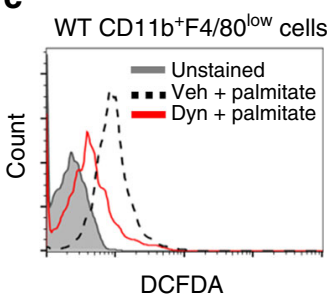

d

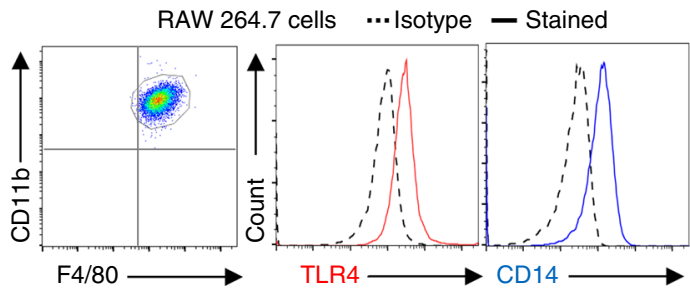

e

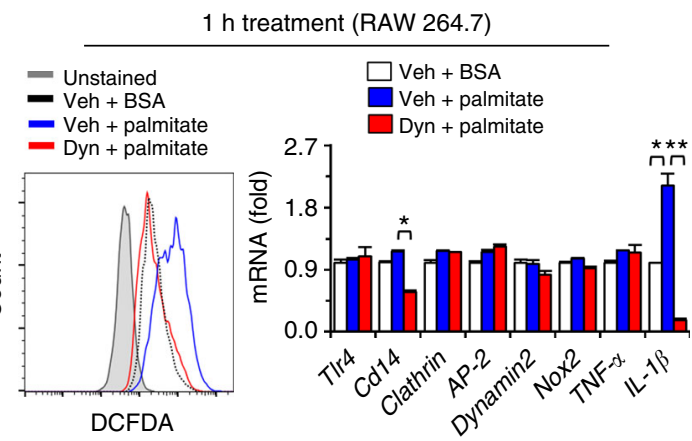

f

f DAPI
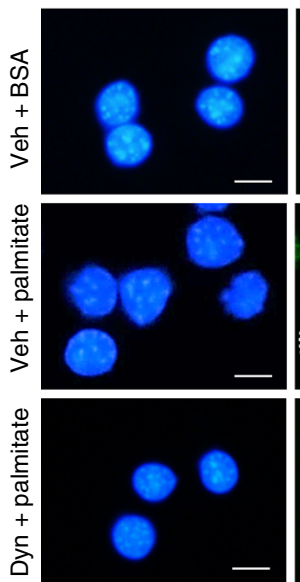

TIr4
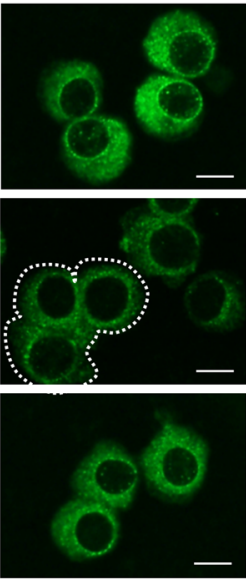

Nox2

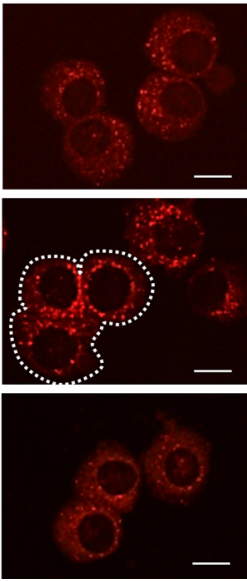

Merge

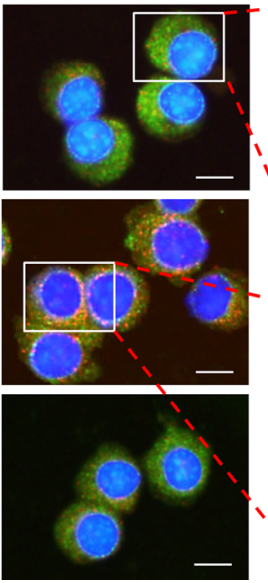

Merge without DAPI

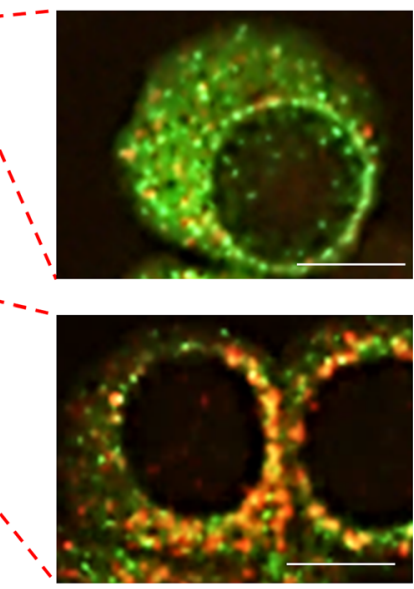

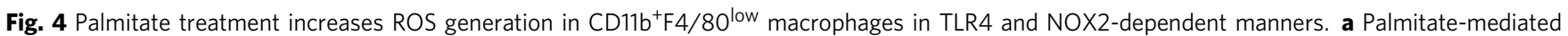
generation of reactive oxygen species (ROS) was monitored by DCF fluorescence in freshly isolated CD11b ${ }^{+} \mathrm{F} 4 / 80^{\text {high }}$ Kupffer cells and CD11b ${ }^{+} \mathrm{F} 4 / 80^{\text {low }}$ macrophages of WT, Nox2 knockout (KO) and TIr4 KO mice. b Freshly isolated macrophages and Kupffer cells of WT mice were subjected to quantitative real-time PCR (qRT-PCR) analyses. $\mathbf{c}$ Isolated macrophages of WT mice were treated with palmitate with or without dynasore (Dyn) treatment. $\mathbf{d}$ RAW 264.7 macrophages were subjected to flow cytometry analyses. e, f RAW 264.7 macrophages were treated with palmitate $(200 \mu \mathrm{M}) \pm$ dynasore $(80 \mu \mathrm{M})$ for $1 \mathrm{~h}$. Then, these cells were subjected to ROS generation assays, qRT-PCR analyses and immunostaining with antibodies of TLR4 and NOX2. Bar $=10$ $\mu \mathrm{m}$. Dotted white line indicates cell boundaries. Solid white rectangles are magnified. Data are representative of three independent experiments in vitro using isolated liver immune cells from 3 (a-c) mice per group. Data are expressed as the mean \pm s.e.m. and analyzed by Student's $t$-test or one-way analysis of variance, ${ }^{\star} P<0.05,{ }^{\star \star} P<0.01$ in comparison with the corresponding controls

(hTLR4-MD2) complex was eluted around the $14 \mathrm{ml}$ fraction compared with controls such as thyroglobulin, $\gamma$-globulin, ovalbumin, myoglobin, and vitamin B12, indicating the formation of a stable monomeric TLR4-MD2 complex ( 90 kDa, 1:1) (Fig. 6a). After incubation with LPS Ra, a significantly forwardshifted peak at the $12 \mathrm{ml}$ fraction was identified as dimerized hTLR4-MD2 complex, whereas the unbound monomeric hTLR4-MD2 complex was eluted at the $14 \mathrm{ml}$ fraction (Fig. 6b). Surprisingly, the hTLR4-MD2 complex incubated with C16BODIPY remained as a monomeric complex (eluted at $14 \mathrm{ml}$ ), despite its tight binding to C16-BODIPY, which was confirmed by fluorescence signal and Commassie staining on Native PAGE (Fig. 6c). To exclude unexpected binding of C16-BODIPY, the
hTLR4-MD2 complex was further incubated with palmitate conjugated with bovine serum albumin (BSA) or BSA only. Consistent with C-16 BODIPY, incubation with palmitate-BSA or BSA did not induce dimerization of the hTLR4-MD2 complex (Fig. 6d, e). These data suggest that unlike LPS binding to a dimerized TLR4-MD2 complex, palmitate binds to a monomeric TLR4-MD2 complex and may initiate the subsequent internalization of the TLR4-MD2 complex along with NOX2 for ROS generation (Fig. 6f).

Endocytosis of the TLR4-MD2 complex generates ROS in human macrophages. Similar to mouse liver, human liver tissue 
a

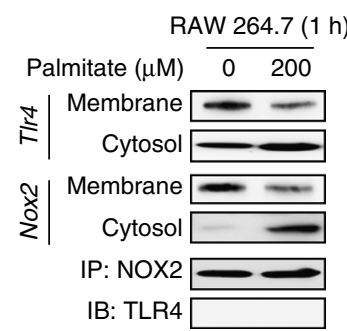

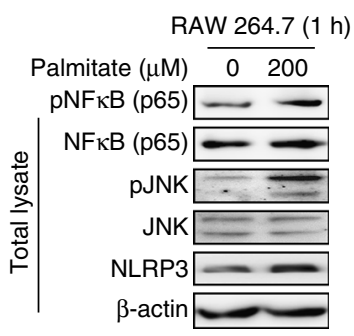

b

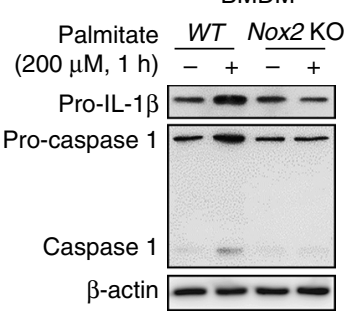

C

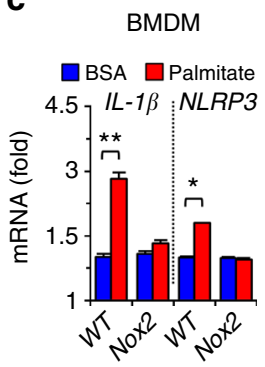

d

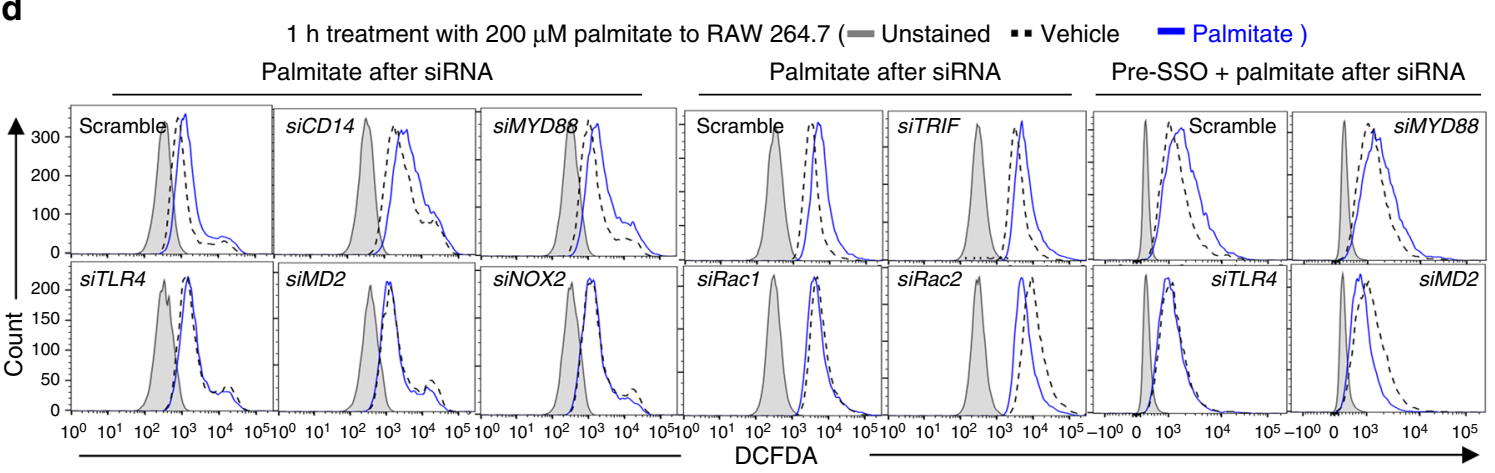

e

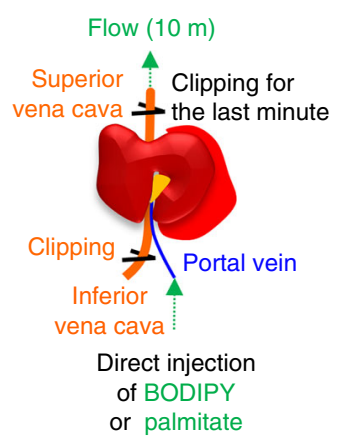

f In vivo palmitate treatment via portal vein $(10 \mathrm{~m})$

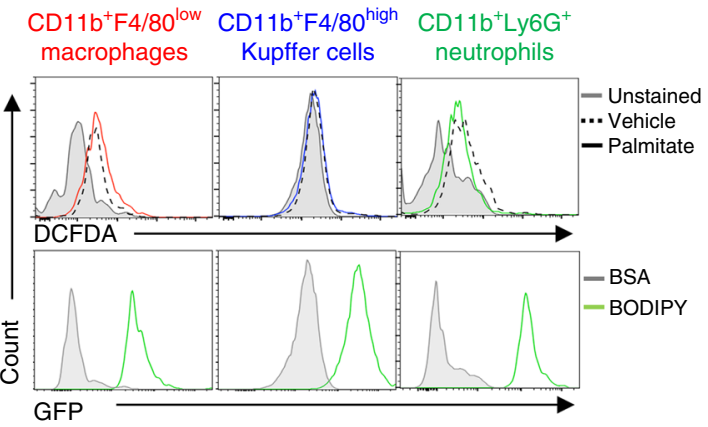

g

$\mathrm{CD} 11 \mathrm{~b}^{+} \mathrm{F} 4 / 80^{\text {low }}$ macrophages after in vivo palmitate treatment $(10 \mathrm{~m})$

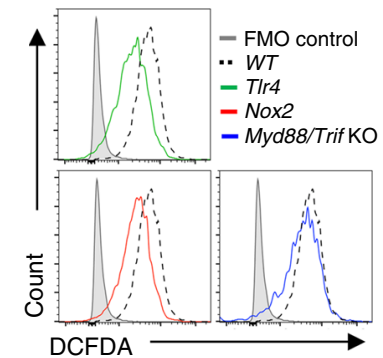

Fig. 5 Endocytosis of palmitate/TLR4-MD2 complex generates NOX2-mediated ROS. a RAW 264.7 cells were stimulated with $200 \mu \mathrm{M}$ palmitate for $1 \mathrm{~h}$. Total cell lysates, membrane fractions or cytosol fractions of RAW 264.7 cells were subjected to Western blotting or immunoprecipitation (IP). With antiNOX2, immunocomplexes from cytosolic fractions were followed by immunoblot analysis with antibody of TLR4. b, c BMDMs of WT and Nox2 KO mice were stimulated with $200 \mu \mathrm{M}$ palmitate for $1 \mathrm{~h}$ and then they were subjected to Western blotting and qRT-PCR analyses, respectively. d RAW 264.7 macrophages were subjected to FACS analyses to measure ROS generation after each siRNA silencing. To inhibit CD36-mediated palmitate uptake, RAW 264.7 cells were pre-treated with sulfosuccinimidyl oleate (SSO) for $10 \mathrm{~min}$. e Direct perfusion of the liver with palmitate. C-16 BODIPY (10 $\mu$ M) and palmitate $(500 \mu \mathrm{M})$ were infused via the portal vein for 10 minutes. Both the superior and inferior vena cava were clipped for the last one minute to trap the solution in the liver. f, $\mathbf{g}$ After perfusing C16-BODIPY or palmitate through the liver of WT, T/r4 KO, Nox2 KO and Myd88/Trif double KO mice, successful delivery of C16-BODIPY and ROS generation were assessed in freshly isolated CD11b $\mathrm{b}^{+} 4 / 80^{\text {low }}$ macrophages, CD11 $\mathrm{b}^{+} \mathrm{F} 4 / 80^{\text {high }}$ Kupffer cells and CD11b ${ }^{+}$Ly6Ghigh neutrophils, respectively. Data are representative of three independent experiments using isolated liver immune cells from 3 (f, $\mathbf{g}$ ) mice per group. Data are expressed as the mean \pm s.e.m. and analyzed by Student's $t$-test, ${ }^{\star} P<0.05,{ }^{\star \star} P<0.01$ in comparison with the corresponding controls

from a patient with $\mathrm{HBV}$-related hepatocellular carcinoma (HCC) also had two types of macrophages such as $\mathrm{CD} 68^{\text {low }} \mathrm{CD} 14^{\text {high }}$ macrophage and $\mathrm{CD} 68^{\text {high }} \mathrm{CD} 14^{\text {low }}$ resident Kupffer cells, whereas only $\mathrm{CD} 68^{\text {low }} \mathrm{CD} 14^{\text {high }}$ monocytes were found in peripheral blood mononuclear cells (PBMCs) from healthy human donor blood (Fig. 7a). Human CD68 ${ }^{\text {low }}$ CD $14^{\text {high }}$ macrophages showed strong ROS production compared with human Kupffer cells (Fig. 7a). Also, CD68 ${ }^{\text {low }} \mathrm{CD} 14^{\text {high }}$ macrophages but not in $\mathrm{CD} 68^{\text {high }} \mathrm{CD} 14^{\text {low }}$ Kupffer cells from normal hepatic tissue sample of the patient with cryptogenic HCC generated ROS in response to palmitate (Supplementary Fig. 6a, b). In addition to combining fatty acid ligand with mTLR4-MD2, mMD2, and hTLR4-MD2 (Fig. 5e; Fig. 6c), hMD2 proteins were co-incubated with C16-BODIPY and the samples were separated by Native PAGE (Fig. 7b). Similarly, hMD2 showed a slight interaction with C16-BODIPY (Fig. 7b; Supplementary Fig. 6c, d). In human $\mathrm{CD} 68^{\text {low }} \mathrm{CD} 14^{\text {high }}$ monocytes isolated from PBMCs, palmitate-induced ROS generation and increased expression of Il-1 $\beta$ and Nlrp3, and these effects were suppressed by dynasore treatment (Fig. 7c). Next, we compared hepatic macrophage population and gene expression of whole liver MNCs isolated from non-tumor/non-steatotic lesions of the patient with cryptogenic HCC and fatty liver lesions of the patient without HCC (Fig. 7d). FACS analyses revealed the same populations of CD $68^{\text {low }}$ CD $14^{\text {high }}$ macrophage and $\mathrm{CD} 68^{\text {high }} \mathrm{CD} 14^{\text {low }}$ Kupffer cells with different frequencies in the two livers, but qRT-PCR analyses revealed significantly increased expression of Tlr4, dynamin2, Rac1, Rac2, Nlrp3, Il-1 $\beta$ and Tnf- $\alpha$ in MNCs from fatty 
a

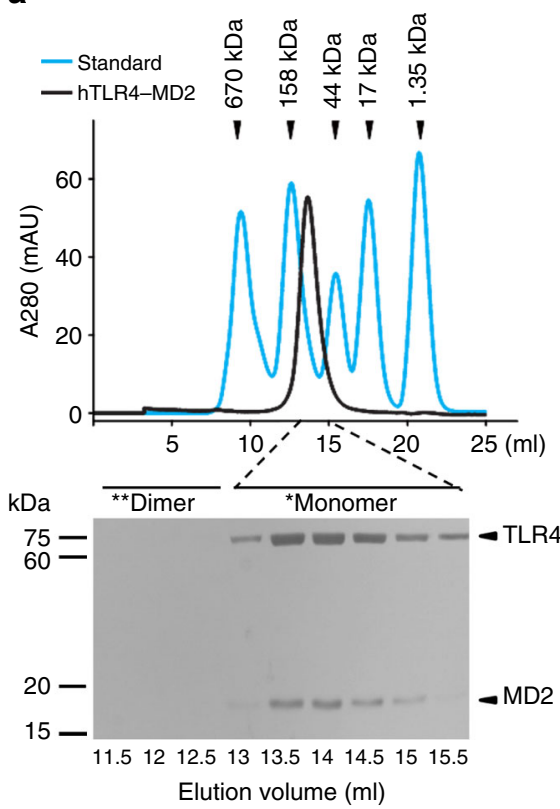

d

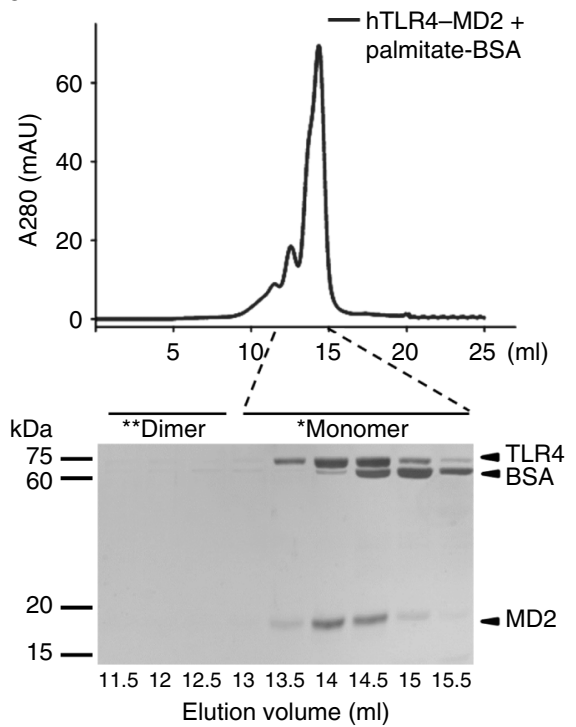

b

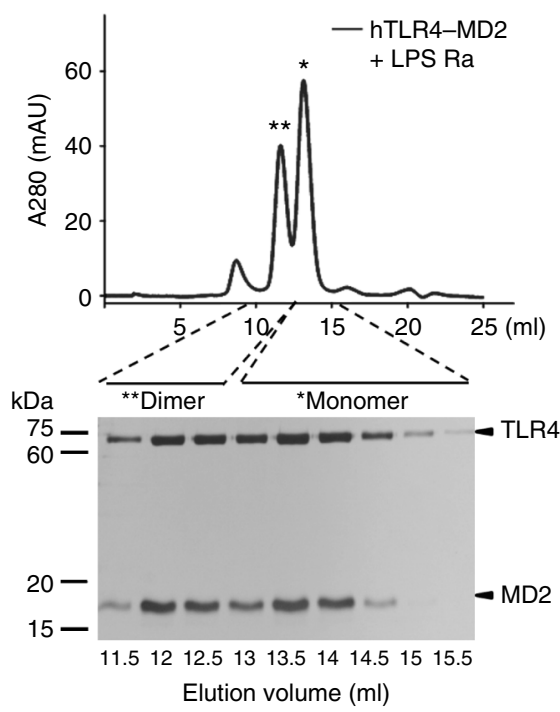

e

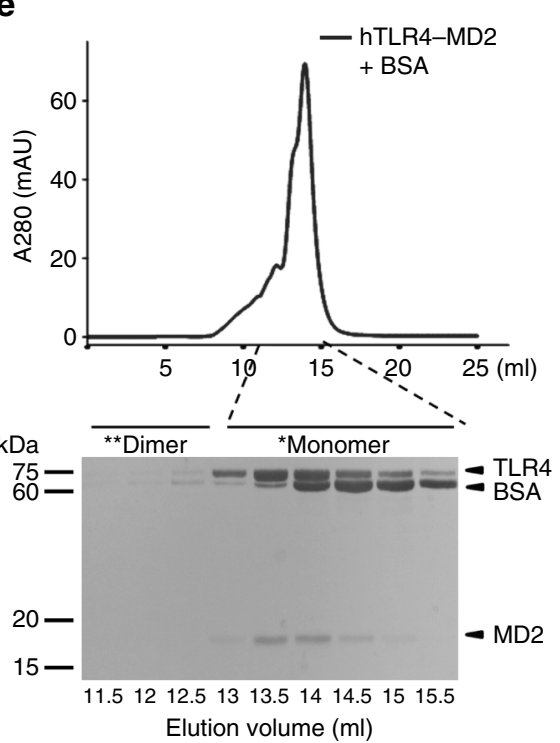

C

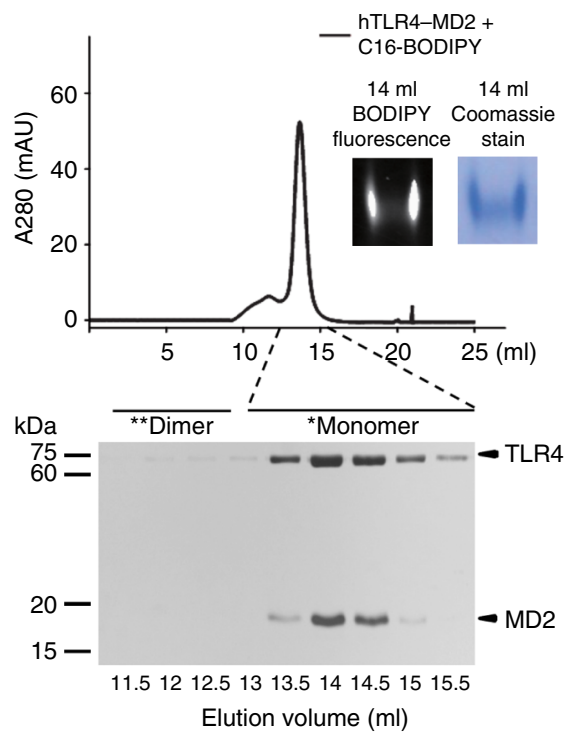

f
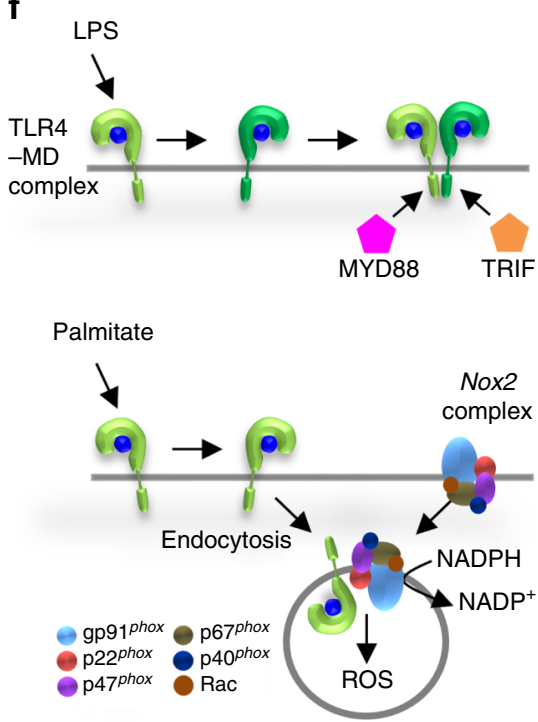

Fig. 6 Monomeric interaction of hTLR4-MD2 complex in response to palmitate treatment. Dimerization of human TLR4-MD2 (hTLR4-MD2) complex mediated by different lipid ligands was examined by size-exclusion chromatography (Superdex 200) and SDS-PAGE with silver staining. a Arrowheads in chromatogram indicate each elution volume of standard proteins (thyroglobulin, $670 \mathrm{kDa}$; -globulin, $158 \mathrm{kDa}$; ovalbumin, $44 \mathrm{kDa}$; myoglobin, $17 \mathrm{kDa}$; vitamin B12, $1.35 \mathrm{kDa}$ ). The monomeric hTLR4-MD2 complexes ( $90 \mathrm{kDa}$ ) were eluted at $\sim 14 \mathrm{ml}$ fraction. $\mathbf{b}$ Incubation of hTLR4-MD2 complex with LPS Ra induced the dimerization of hTLR4-MD2 complex. The dimeric hTLR4-MD2 complex by LPS was eluted at 12 ml and the remaining monomeric TLR4-MD2 complexes were eluted at $14 \mathrm{ml}$. c-e Incubation of hTLR4-MD2 complex with C16-BODIPY (c), palmitate-BSA (d), or BSA (e) could not induce the dimerization of hTLR4-MD2 complexes. Native PAGE followed by illumination at $488 \mathrm{~nm}$ and Coomassie staining confirmed the binding of C16BODIPY to hTLR4-MD2 complex (c, inset). f Schematic diagram of dimeric or monomeric states of TLR4-MD2 complex in response to treatments of LPS and palmitate. The dimeric hTLR4-MD2 complex by LPS requires binding with MyD88 or TRIF, while monomeric TLR4-MD2 complex by palmitate generates ROS by NOX2 complex. Data are representative of three independent experiments

liver compared to MNCs from non-steatotic normal lesion of HCC (Fig. 7d). Moreover, in a data set downloaded from the GEO database (accession code GSE63067), gene expression of Tlr4, Nox2, Rac1 and Rac2 was remarkably increased in patients with non-alcoholic steatohepatitis compared with heathy controls (Supplementary Fig. 7). These data suggested that endocytosis of TLR4-MD2 complex in human $\mathrm{CD} 68^{\text {low }} \mathrm{CD} 14^{\text {high }}$ macrophages by palmitate-mediated ROS generation, which might be related with pathogenesis of NAFLD in human patients.
NOX2-deficient BM transplantation attenuates hepatic steatosis. In order to exclude off-target effects of NOX2 and to confirm the importance of NOX2 in macrophages, we generated chimeric mice by reciprocal bone marrow transplantation (BMT) using $W T$ and Nox2 KO mice $\left(W T^{W T}, W T^{N O X 2}, N O X 2^{W T}\right.$, or $N O X 2^{N O X 2}$ ) as previously reported (Supplementary Fig. 8a ${ }^{22}$. Body, liver and epididymal fat pad weights were significantly lower in mice with Nox2 KO BMT (WT NOX2 and NOX2NOX2 than in mice with WT BMT $\left(W T^{W T}\right.$ and $\left.N O X 2^{W T}\right)$ 
a

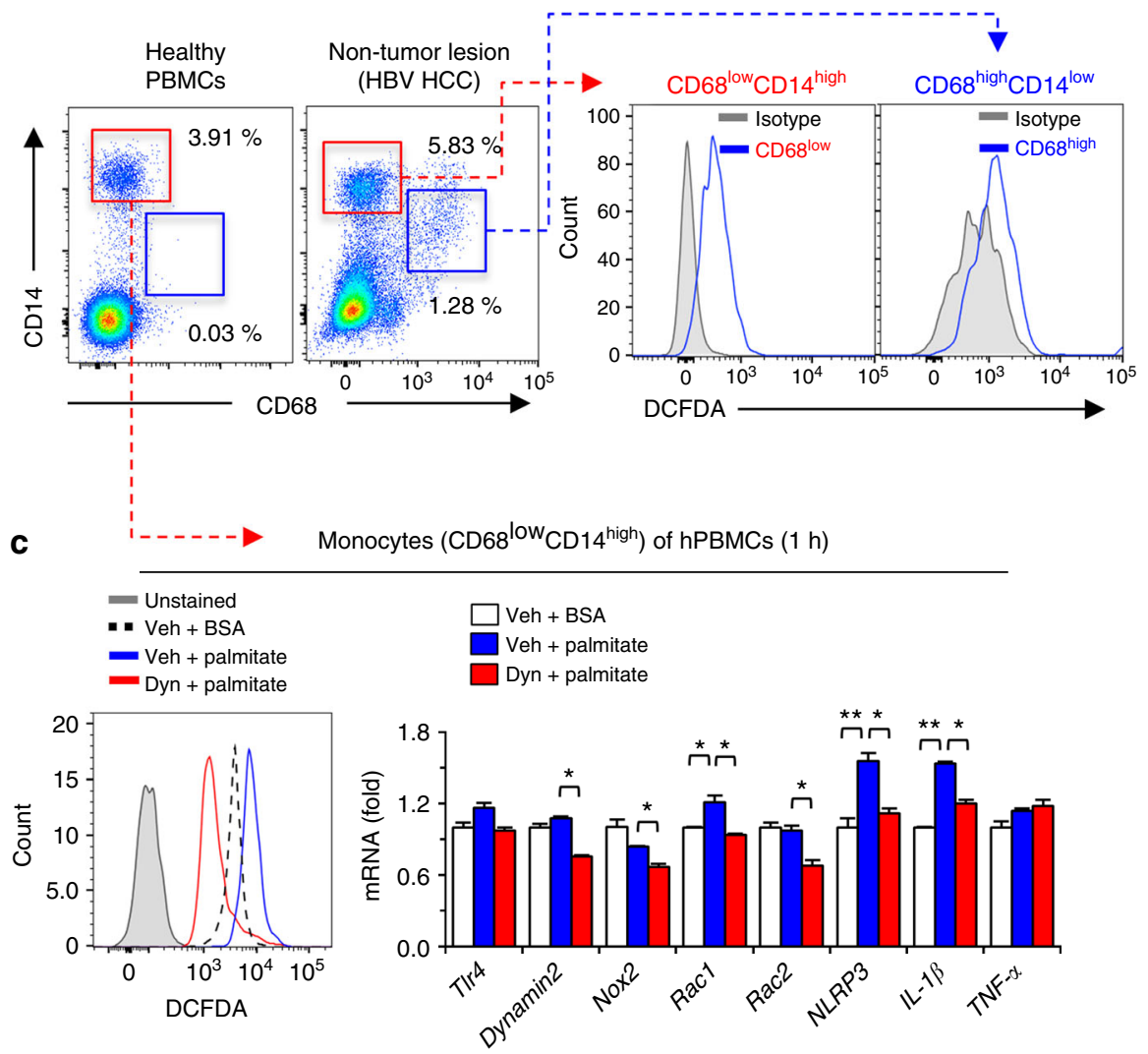

b

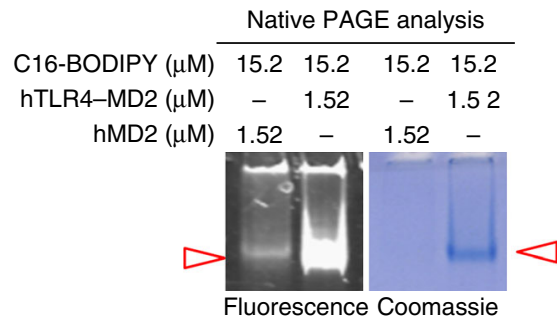

d

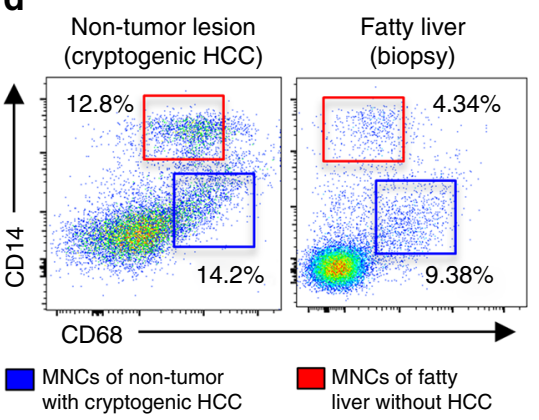

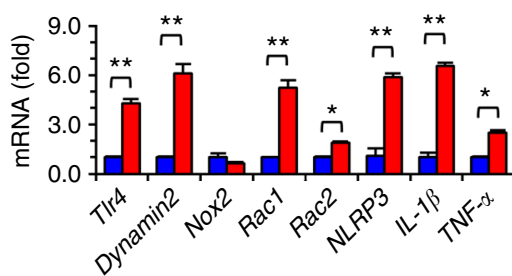

Fig. 7 Palmitate treatment increases ROS generation in human CD68 ${ }^{\text {low }} C D 14^{\text {high }}$ monocytes via endocytosis of palmitate/TLR4 complex. a Healthy human peripheral blood mononuclear cells (PBMCs) and isolated liver mononuclear cells (MNCs) from non-tumor lesions of HCC (HBV origin) were subjected to flow cytometry and assessment of ROS generation, respectively. $\mathbf{b}$ After the reaction between each protein and BODIPY-labeled fluorescent fatty acid analog (C16-BODIPY) under the indicated conditions, the samples loaded at Native gradient PAGE (4-15\%) were visualized with illumination $(488 \mathrm{~nm})$ and Coomassie staining. c Healthy human PBMCs were treated with palmitate \pm dynasore. Then, these cells were subjected to assessment of ROS generation and qRT-PCR analyses. $\mathbf{d}$ Freshly isolated liver MNCs from non-tumor liver lesions of primary HCC and biopsy lesions of fatty liver were subjected to flow cytometry and qRT-PCR analyses. Data are representative of three independent experiments using PBMC of healthy controls $(n=5)$ and liver MNCs of HBV $(n=3)$, cryptogenic HCC $(n=1)$ and fatty liver $(n=1)$ patients $\mathbf{a}, \mathbf{c}, \mathbf{d}$. Data are expressed as the mean \pm s.e.m. and analyzed by Student's $t$-test or one-way analysis of variance, ${ }^{\star} P<0.05,{ }^{\star \star} P<0.01$ in comparison with the corresponding controls

(Supplementary Fig. 8b, c). WT $T^{\mathrm{NOX} 2}$ and $\mathrm{NOX} 2^{\mathrm{NOX} 2}$ mice had improved glucose tolerance as assessed by GTT (Fig. 8a) and lower serum ALT and TC, as compared to WT NOX2 and $N O X 2^{N O X 2}$ mice (Supplementary Fig. 8d). The livers of $W T^{\text {NOX2 }}$ and $\mathrm{NOX}_{2}{ }^{\mathrm{NOX} 2}$ mice were less yellowish in color and had lower TG content than $W T^{W T}$ and NOX2 ${ }^{W T}$ mice (Fig. 8b). Coincidently, fat accumulation was remarkably reduced, whereas deposition of glycogen was greatly increased in liver sections of $W T^{\mathrm{NOX} 2}$ and NOX2 ${ }^{\mathrm{NOX} 2}$ mice (Fig. $8 \mathrm{c}$ ). Although there were no differences in frequencies of hepatic macrophages among the groups, Il-1 $1 \beta$ and Tnf- $\alpha$ gene expression was considerably decreased whereas Il-10 expression was increased in liver MNCs of $W T^{N O X 2}$ and NOX2 ${ }^{N O X 2}$ mice compared with those of $W T^{W T}$ and $N O X 2^{W T}$ mice (Fig. 8d; Supplementary Fig. 8e, f). Immunoblots of phosphoproteins revealed increased phosphorylation of AMPK and AKT but decreased phosphorylation of NF-KB and JNK in liver tissues of $W T^{\mathrm{NOX} 2}$ and $\mathrm{NOX} 2^{\mathrm{NOX} 2}$ mice compared with those of $W T^{W T}$ and $N O X 2^{W T}$ mice (Fig. 8e). In addition, expression of SREBP1 and FAS was significantly down-regulated in $W T^{N O X 2}$ and $N O X 2^{N O X 2}$ mice (Fig. 8e).

\section{Discussion}

The role of NOX2 on innate immune responses has been well described in mice and humans, yet the mechanism of palmitate-mediated ROS generation via NOX2 is not well understood, especially in transmigrating macrophages in the steatotic and insulin resistant liver. In the present study, we have demonstrated that palmitate binds a monomeric TLR4-MD2 complex in $\mathrm{CD} 11 \mathrm{~b}^{+} \mathrm{F} 4 / 80^{\text {high }}$ and $\mathrm{CD} 68^{\text {low }} \mathrm{CD} 14^{\text {high }}$ macrophages of mice and humans, respectively, which leads to NOX2-mediated ROS generation by dynamin-mediated endocytosis of the TLR4-MD2 complex. Another striking finding was that the HFD or palmitateinduced ROS generation and pro-inflammatory changes selectively appeared in transmigrating liver macrophages and not in resident Kupffer cells. This, in turn, is responsible for the obesity-related steatohepatitis and insulin resistance, as summarized in Fig. $8 \mathrm{f}$.

Increased inflammation is a key feature of metabolic disorders such as hepatic steatosis and insulin resistance. Recent studies have indicated that genetic depletion of NOX2 resulted in reduced visceral adiposity, diminished visceral fat macrophage infiltration and improved glucose regulation in HFD-fed male mice $^{23}$, and that a deficiency in the cytosolic NOX2 system component p47phox resulted in reduced hepatic TG in HFD-fed male and female mice ${ }^{24}$. In addition, Ezetimibe-mediated reduction of NOX2 activation attenuated hepatic steatosis and macrophage infiltration of $\mathrm{db} / \mathrm{db}$ mice ${ }^{25}$. These findings suggest that NOX2 plays an important role in diet-induced obesity, hepatic steatosis and insulin signaling. In contrast, singly housed 
a

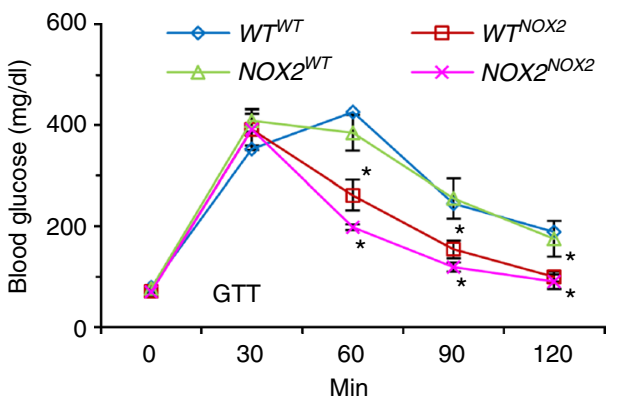

C
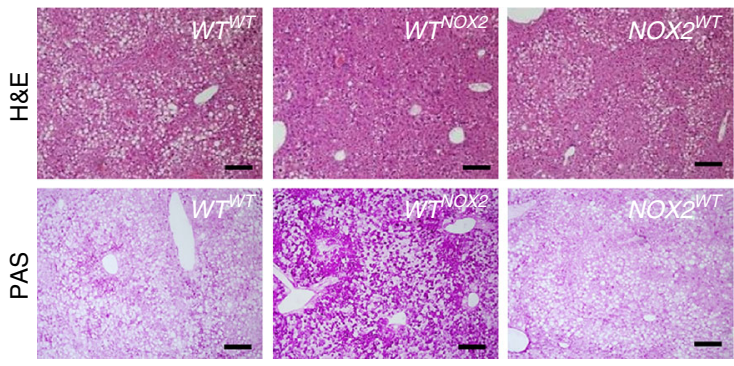

e

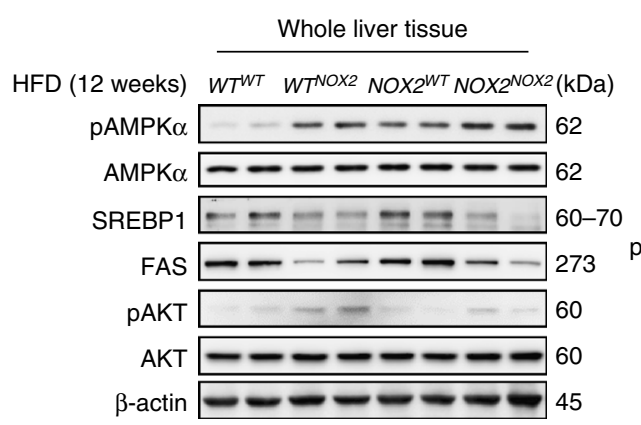

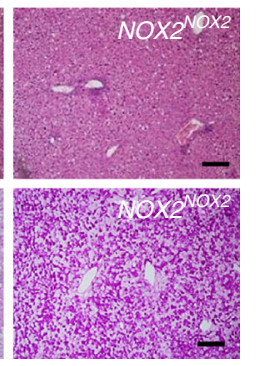

b
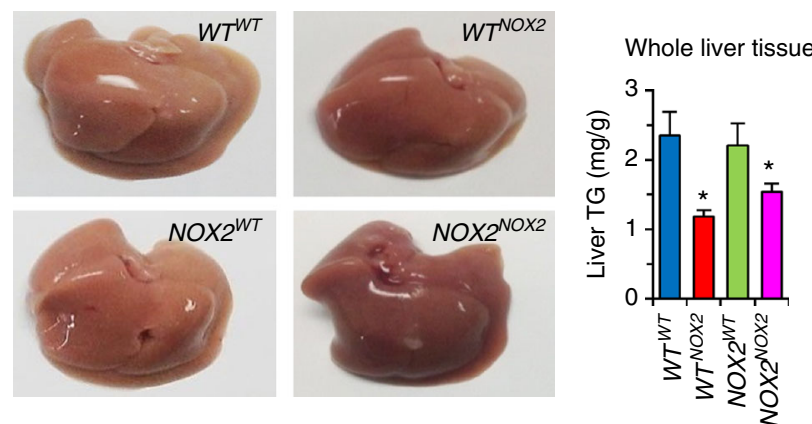

d

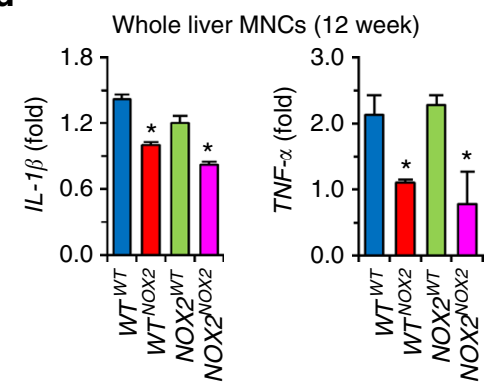

Fig. 8 NOX2-deficient bone marrow transplantation attenuates high-fat diet-induced hepatic steatosis in mice. After reciprocal bone marrow transplantation between WT and Nox2 KO mice, mice were fed a high-fat diet for 12 weeks. a GTT was performed after $16 \mathrm{~h}$ fasting. $\mathbf{b}$ Liver gross findings and hepatic contents of TG were assessed. c Liver sections were stained with H\&E (upper panels) and PAS (lower panels). Bar $=100 \mu \mathrm{m}$. $\mathbf{d}$ Isolated liver MNCs were subjected to qRT-PCR analyses. e Whole liver tissues were subjected to Western blottings. f Schematic diagram of NOX2-mediated ROS generation through endocytosis of monomeric TLR4-MD2 complex in non-resident macrophages by palmitate and its involvement in the pathogenesis of hepatic steatosis and insulin resistance. Data are representative of two independent experiments using 5 mice per group. Data are expressed as the mean \pm s.e.m. and analyzed by one-way analysis of variance, ${ }^{\star} P<0.05$ in comparison with the corresponding controls

Nox2 KO mice maintained on HFD from weaning for up to 18 weeks had less epididymal white adipose tissue but more hepatic lipid accumulation, inflammatory signatures and insulin resistance than $W T$ mice ${ }^{26}$. One should note, however, that social isolation stress in single housed mice has been shown to induce liver hypertrophy and an upregulation of lipid synthetic and downregulation of lipid oxidation pathways ${ }^{27}, 28$, which complicates interpretation of the data in the above study ${ }^{26}$. Through the use of reciprocal BMT, our study clearly showed improved glucose sensing, less fat but more glycogen accumulation in hepatocytes and attenuated inflammatory responses in $W T$ and Nox2 KO mice with Nox2 KO BMT compared with WT and Nox2 KO mice with WT BMT (Fig. 8; Supplementary Fig. 8). In addition, only $\mathrm{CD} 11 \mathrm{~b}^{+} \mathrm{F} 4 / 80^{\mathrm{low}}$ macrophages generated ROS in response to in vivo perfusion with palmitate, although Nox2 was expressed

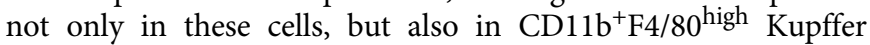

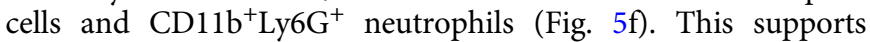
that beneficial effects of NOX2-depleted BMT on high-fat diet-induced hepatic steatosis and insulin resistance might be due to less ROS generation of transmigrating macrophages. Using a similar BMT approach, another study demonstrated that WT mice with Nox2 KO BMT had decreased ROS production in mononuclear cells and impaired neovascularization following hindlimb ischemia ${ }^{29}$. However, further studies using macrophage-specific conditional Nox2 KO mice are needed to provide more definitive evidence for this hypothesis.

In addition to LPS, TLR4 can bind a broad range of ligands including saturated FFAs, which may lead to diverse signaling complexes due to the possible heterodimerization of TLR4 with other TLRs, co-receptors or accessory proteins including CD14, CD36 and MD2 $2^{14,30,31}$. Heterodimerization between TLR4 and TLR2 or TLR4 and TLR6 has been reported in microglial cells and macrophages, in which either heterodimers of TLR4-TLR 2 by hemoglobin or TLR4-TLR6 by oxidized LDL/amyloid- $\beta$ triggered inflammatory responses in a MyD88-dependent manner ${ }^{30}, 31$. In contrast, we found that MyD88 and TRIF were not required 
for palmitate-induced ROS generation in vitro and in vivo (Fig. 5d-g). Similarly, minimally oxidized LDL treatment generated NOX2-mediated ROS in peritoneal macrophages by a TLR4-dependent, MyD88-independent manner ${ }^{11}$. In cases of CD14-dependent endocytosis of TLR4, LPS-mediated dimerization of the TLR4-MD2 complex is essential, in which CD14, Syk and PLC 2 play critical roles, whereas TLR4 signaling is not necessary for TLR4 endocytosis in dendritic cells and bone marrow-derived macrophages 32,33 . Our data show that the TLR4-MD2 complex was required, whereas CD14 and CD36 were dispensable for the palmitate-induced, NOX2-mediated ROS production (Fig. 5d). Furthermore, unlike LPS, palmitate binds the momomeric TLR4-MD2 complex (Fig. 6), which is a novel type of signaling complex of palmitate-TLR4-NOX2 in macrophages.

Some reports suggested that activation of TLR2 or TLR4 signaling might be attributed to endotoxin contamination in dietary saturated fatty acids ${ }^{14,34}$. However, in contrast to LPS, dimerization of the TLR4-MD2 complex was not observed in treatments with C16-BODIPY, palmitate-BSA or even BSA, which excludes the role of contamination of endotoxins (Fig. 6). Similar to the dynamin-mediated endocytic pathway of LPSTLR $4^{15}$, internalization of TLR4-MD2 complex was also dynamin dependent in both mouse macrophages and human monocytes, as indicated by the suppression of palmitate-TLR4-NOX2mediated ROS generation by dynasore, an inhibitor of dynamin (Fig. 4e; Fig. 7c). Endocytosis by dynamin also plays important roles in NOX2-mediated ROS generation in meningococcal endotoxin-treated neutrophils and hypoxia-stimulated endothelial cells, respectively ${ }^{35}, 36$. We further demonstrated that palmitate strongly binds with monomeric TLR4-MD2 complex and MD2 protein in mouse and human (Fig. 6; Supplementary Fig. 5; Fig. 7b). Our findings are in agreement with those in a recent study demonstrating direct and dose-dependent binding of palmitate with protein MD2 to activate TLR4 signaling in thioglycollate-induced peritoneal macrophages ${ }^{37}$.

Regarding activation of NOX2 after binding TLR4 with palmitate, either Tlr4 knockdown or Nox2 deletion remarkably reduced palmitate-induced ROS, suggesting a reciprocal interplay between NOX2 and TLR4 (Fig. 4a). There is ample evidence that in response to diverse stimuli, clustering of lipid rafts (LRs) in the plasma membrane drives NOX assembly and aggregation, and LPS stimulates the association of TLR4 and its accessory proteins with LRs, triggering a signal cascade ${ }^{38-40}$. Our data indicate that the TLR4 and NOX2 proteins do not co-localized in the plasma membrane under resting conditions, but display increased colocalization, albeit not direct binding, following palmitate treatments as they translocated to the perinuclear area (Fig. 4f; Fig. 5a). This suggests that TLR4 bound by palmitate might induce clustering of LRs, which are also enriched in components of the NOX2 complex, leading to the internalization of these molecules to generate ROS. However, further studies are needed to elucidate the exact mechanism involved.

Interesting studies have suggested that in vivo and in vitro HFD feeding and treatments with palmitate prime NLRP3 inflammasome via TLR2- and TLR4-mediated NF- $\kappa B$ signaling pathways in macrophages, dendritic cells and Kupffer cells to upregulate expression of $I l-1 \beta$ and $N l r p 3$, leading to peripheral inflammation, obesity, hepatic steatosis and insulin resistance ${ }^{41-}$ 43. Similarly, NOX activation contributes to NLRP3 inflammasome activation. In LPS-primed human and mouse macrophages, NOX4-mediated increased expression of carnitine palmitoyltransferase 1A promotes NLRP3 inflammasome activation by fatty acid oxidation-induced mitochondrial ROS generation ${ }^{17}$. In addition, NOX2 is necessary for palmitate-induced NLRP3 inflammasome activation in endothelial cells ${ }^{44}$. These reports suggest that NLRP3 inflammasome activation might be related to activation of TLRs and NOXs. However, all priming-mediated ROS generation and inflammatory responses in macrophages were assessed at later time points (more than $6 \mathrm{~h}$ ) after palmitate treatment, whereas NOX2-mediated ROS production plateaued at 45 to $60 \mathrm{~min}$ in macrophages, and RAW 264.7 cells $^{35}$, 45. Thus, in the present study, we assessed early changes of ROS production and gene expression in human and mouse macrophages including RAW 264.7 cells at $1 \mathrm{~h}$ after palmitate treatment. ROS production, activation of NF- $\kappa \mathrm{B}$ and JNK, and increased expression of $\mathrm{Il}$ $1 \beta$ and Nlrp3 mRNA were detected within $1 \mathrm{~h}$ of palmitate treatment, and these early effects were clearly independent of MyD88 and TRIF (Fig. 5; Supplementary Fig. 4) and could be reversed by depletion of NOX2 and TLR4 or dynasore treatment (Fig. 4e; Fig. 5b, c; Supplementary Fig. 4b, c; Fig. 7c). Furthermore, in vivo intraportal perfusion with palmitate resulted in increased ROS generation only in $\mathrm{CD} 11 \mathrm{~b}^{+} \mathrm{F} 4 / 80^{\text {low }}$ macrophages in a TLR4/NOX2-dependent, MyD88/TRIF-independent manner, although NOX2 was highly expressed in macrophages, Kupffer cells and neutrophils in the liver (Fig. 5f, g). All these findings suggest that early priming of NLRP3 inflammasome by palmitate-TLR4-NOX2-mediated ROS in macrophages enhances further inflammatory stress signals including LPS-mediated canonical signaling of TLR4 at later phase or amplifies inflammatory responses of macrophages and other immune cells (e.g., Kupffer cells) by either an autocrine or paracrine manner.

According to the literature ${ }^{2,3}$, Kupffer cells and macrophages both support the process of inflammation and insulin resistance in liver. However, our study clearly shows that rather than CD11b

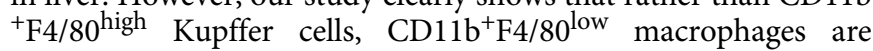
responsible for NOX2-dependent ROS production in response to HFD-mediated liver injury and palmitate treatment (Fig. 2c; Fig. 4a). In addition, CD $11 b^{+} F 4 / 80^{\text {high }}$ Kupffer cells did not generate ROS in response to palmitate (Fig. 4a). Although we did not investigate the underlying mechanism, the inability of palmitate to induce dynamin expression maybe a factor. Nevertheless, our data may explain why enriched Kupffer cells in liver show resistance to elevated circulating free fatty acids after HFD feeding to mice.

In addition to liver, NOX2 complex including NOX subunit proteins (e.g. p47phox) are widely expressed in multiple organs and are involved in the pathogenesis of infectious diseases. LPSmediated NOX2 activation has been suggested in pulmonary vascular endothelial cells and cardiac fibroblast, subsequently leading to lung inflammation and cardiac fibrosis, respectively 46 , 47. Similarly, Nox2 deletion in muscle tissue alleviates insulin resistance by reducing oxidative stress in HFD-fed mice ${ }^{48}$. Moreover, Nox2 deletion in apolipoprotein E KO mice reduces atherosclerotic plaque development and plasma lipids by decreasing ROS in aortic endothelial cells and macrophages of HFD-fed mice ${ }^{49}$. Furthermore, palmitate treatment induced dysfunction and apoptosis of pancreatic $\beta$ cells via NOX2mediated $\operatorname{ROS}^{50}$. On the contrary, in response to mycobacteriainduced TLR2 activation, Nox2 depletion in macrophages prominently abrogated antimicrobial activity by decreasing production of cytokines and chemokines ${ }^{51}$.

In summary, our findings shed new light on the pathophysiologic mechanisms of TLR4 and NOX2. We have demonstrated an interplay between palmitate and monomeric TLR4-MD2 complex in mediated ROS generation selectively in macrophages but in Kupffer cells, via dynamin-dependent endocytosis of NOX2 and TLR4, and the key role of this signaling in promoting hepatic steatosis and insulin resistance in NAFLD. Therefore, our findings imply that endocytosis of TLR4 and NOX2 could be a novel therapeutic target for NAFLD, including hepatic steatosis and insulin resistance. 


\section{Methods}

Mice. Male C57BL/6 based WT, Nox2 (gp91 ${ }^{\text {phox }}$ ) and Trl4 KO mice were purchased from Jackson Laboratories (Bar Harbor, ME, USA), MyD88 KO and Trif KO mice on C57BL/6 background were provided by Dr. Shizuo Akira, and MyD88/ Trif double KO mice were kindly generated by Professor Suk-Jo Kang. These mice were maintained on a regular light-dark cycle (12-hour light/12-hour dark) at $24^{\circ} \mathrm{C}$ with $40-60 \%$ humidity in a specific pathogen-free animal facility at Korea Advanced Institute of Science and Technology (KAIST, Daejeon, Republic of Korea). All experimental protocols including ethics were approved by the institutional Animal Care and Use Committee (IACUC) of KAIST (No. KA2011-40). The 8-week-old male mice (5-8 mice) were fed ad libitum with normal chow diet (fat content ranges from 4 to 6\%) or high-fat diet (Harlan Teklad, TD 06414, Madison, WI, USA) which approximately $60 \%$ of total calories came from fat for 12 weeks. Mice with arthritis, which occasionally occurred in NOX2 mice, were ejected from test group. Body weight and diet intake were measured twice a week during this period.

Intraportal perfusion with palmitate. To circulate palmitate throughout the liver, hepatic blood was flushed out by brief perfusion with a buffer solution (1× PBS with antibiotics) through the portal vein of anesthetized WT, Tlr4 KO, Nox2 KO and $M y D 88 /$ Trif double $\mathrm{KO}$ mice. Then, $10 \mathrm{ml}$ of a solution of $500 \mu \mathrm{M}$ palmitate or $10 \mu \mathrm{M}$ C16-BODIPY was infused for $10 \mathrm{~min}$. For the last one minute, both the superior and inferior vena cava were clipped, trapping the solution in the liver. Next, the extracted liver was dissociated into single cells through Gentle MACS/ Mouse liver dissociation kit (Miltenyi Biotec, Bergisch Gladbach, Germany). Isolated cells were stained with antibodies including DCF-DA and surface antibodies. Production of ROS was measured immediately by flow cytometry (LSRII, BD Bioscience, San Jose, CA, USA).

Bone marrow transplantation. Chimeric mice were generated by reciprocal bone marrow transplantation. Mice were irradiated with single dose $9 \mathrm{~Gy}$ and then were reconstituted with bone marrow cells $\left(2 \times 10^{6}\right.$ per mouse) of femur and tibias of donor mice through tail vein injection. After allowing to rest 8 weeks, chimerism was confirmed by isolated liver MNCs of mice (Supplementary Fig. 8a).

Serum biochemical measurements. Serum was collected from blood and assayed for levels of alanine aminotransferase (ALT), aspartate aminotransferase (AST), triglyceride (TG), and total cholesterol (TC) using VET Test Chemistry Analyzer according to manufacturer's instruction (IDEXX Laboratories, Westbrook, ME, USA).

Histological analyses. Parts of left and medial lobes were fixed with $10 \%$ neutral buffered formalin or prepared using the Tissue-Tek OCT compound (Sakura, Tokyo, Japan). After deparaffinization and rehydration, paraffin sections with $5 \mu \mathrm{m}$ thickness were stained with hematoxylin and eosin (H\&E), or Periodic Acid-Schiff (PAS) reagents (Sigma-Aldrich, St. Louis, MO, USA), whereas frozen sections with $10 \mu \mathrm{m}$ thickness were subjected to oil-red O staining (Sigma-Aldrich, St. Louis, MO, USA) for visualization of lipid droplets.

Liver TG levels. Hepatic lipids were extracted from $50-100 \mathrm{mg}$ of liver tissues using chloroform/methanol mixture (2/1 ratio). Lipid extract was lyophilized by nitrogen gas and then dissolved in 5\% fatty acid-free BSA. Resuspended lipid was used for assessments of TG level using VET Test Chemistry Analyzer according to the manufacturer's instructions (IDEXX Laboratories, Westbrook, ME, USA).

Glucose tolerance test and insulin tolerance test. For the glucose or insulin tolerance test, the mice were fasted from 18:00 PM to 10:00 AM (16 h) or 8:00 AM to 12:00 PM $(4 \mathrm{~h})$, respectively, and allowed free access to water. Glucose $(2 \mathrm{~g} \mathrm{~kg}$ ${ }^{-1}$; Sigma-Aldrich St. Louis, MO, USA) or $1 \mathrm{U} \mathrm{kg}^{-1}$ insulin (Humalog, Lilly, Indianapolis, IN, USA) was injected intraperitoneally to mice. Tail blood glucose levels were measured by using a glucometer (Allmedicus, Anyang, South Korea) at $0,30,60,90,120$ and $150 \mathrm{~min}$ after glucose injection or at $0,15,30,45,60,90$, and $120 \mathrm{~min}$ after insulin injection.

siRNA transfection. Target cells were transfected with $20 \mathrm{nM}$ of siRNA against Nox2, Tlr4, Rac1, Rac2, Myd88, Trif, Md2, and Cd14 (Bioneer, Daejeon, Korea; Supplementary Table 1) using the Lipofectamine RNAiMAX transfection reagent (ThermoFisher Scientific, Waltham, MA, USA) according to the manufacturer's instructions. After determining knockdown efficiency by quantitative RT-PCR at $24 \mathrm{~h}$-transfection, experiments were performed.

Quantitative RT-PCR. Total RNA was isolated from liver tissues or cells with TRIzol reagent (Thermo Fisher Scientific, Waltham, MA, USA) or RNeasy Mini kit (Qiagen, Hilden, Germany) in accordance of the manufacturer's instructions. The same quantity of total RNA was reverse-transcribed to cDNA using amfiRivert cDNA synthesis master mix (GenDEPOT, Houston, TX, USA). Quantitative RT-PCR was performed by SYBR Green Real-time PCR Master Mix (Toyobo,
Osaka, Japan). To quantify transcription, the mRNA expression levels of the target genes were normalized to $\beta$-actin or $18 \mathrm{~S}$. The primers used in this study were listed in Supplementary Table 2. All samples were run in duplicate and the relative gene expression calculated as $2^{-\Delta \mathrm{CT}}$ was expressed as fold increased over control samples.

Western blotting. Total protein samples were isolated from frozen liver tissue and cultured cells using RIPA lysis buffer ( $30 \mathrm{mM}$ Tris, $\mathrm{pH} 7.5,150 \mathrm{mM} \mathrm{NaCl}, 1 \mathrm{mM}$ PMSF, $1 \mathrm{mM} \mathrm{Na}_{3} \mathrm{VO}_{4}, 10 \%$ SDS, $10 \%$ glycerol), containing protease and phosphatase inhibitors (Roche, Basel, Switzerland). Samples were separated in a $10 \%$ SDS-polyacrylamide gel electrophoresis and transferred onto nitrocellulose membrane. Membranes were blocked in 5\% milk, incubated with primary antibodies at 1:1000 in PBST. pAMPK $\alpha$ (\#2535), AMPK $\alpha$ (\#2532), FAS (\#3180), pAKT (\#9271), AKT (\#9272), pNF-кBp65 (\#3033), NF-кBp65 (\#4764), pJNK (\#9251), JNK (\#9252), Caspase-1 (\#2225), IL-1ß (\#12507), NLRP3 (\#15101) and $\beta$-actin (\#4970) (Cell Signaling Technology, Danvers, MA, USA), SREBP1 (\#MA5-16124) (US Biological Life Science, Marblehead, MA, USA) and TLR4 (\#SC-293072) (Santa Cruz Biotechnology, CA, USA). Comparative amount was normalized with $\beta$-actin. According to the manufacturer's instructions, membrane proteins were isolated by using the membrane protein isolation kit (Thermo Fisher Scientific, Waltham, MA USA) and the samples underwent for co-immunoprecipitation assays (Co-IP). CoIP was performed using a commercial kit Pierce ${ }^{\mathrm{TM}}$ Co-Immunoprecipitation Kit (Thermo Fisher Scientific, MA, USA) In brief, proteins cross-linked to target proteins were immune-precipitated with $10 \mu \mathrm{g}$ anti-NOX2 antibody (\#SC-130543) (Santa Cruz Biotechnology, CA, USA). After this cross-reaction, mixtures incubated with protein A/G PLUS agarose beads (\#SC-2003) (Santa Cruz Biotechnology, CA, USA). The immunoprecipitated proteins were isolated by boiled sample buffer and were followed by $10 \%$ SDS-PAGE.

Primary macrophage isolation. To isolate infiltrating macrophages and resident Kupffer cells in liver, three different methods were used. All resuspended cells were further sorted by MACS (Miltenyi Biotec, Bergisch Gladbach, Germany), Magnisort (Affymetrix, Santa Clara, CA, USA) or FACS Aria II (BD Bioscience, San Jose, CA, USA) by using antibodies of APC-Cy7 tagged anti-mouse CD11b (clone M1/ 70) and PE-Cy7 conjugated anti-mouse F4/80 (Clone BM8) (BD pharmingen and affymetrix). First, for isolation of infiltrated macrophages, mouse liver mononuclear cells (MNCs) were isolated with density gradient Percoll solution (GE Healthcare Life Science, Chicago, IL, USA). In brief, mouse livers were homogenized and filtered through a $70 \mu \mathrm{m}$ nylon cell strainer (BD Bioscience, San Jose, CA, USA). After removing debris, hepatic non-parenchymal cells were collected and suspended in $40 \%$ Percoll. The cell suspension was gently overlaid onto $70 \%$ Percoll and centrifuged at $4{ }^{\circ} \mathrm{C}$ for $25 \mathrm{~min}$ at 3000 r.p.m. Liver MNCs were collected from the interface. Then, liver MNCs were resuspended in fresh PBS after $\mathrm{RBC}$ lysis. Second, to get resident Kupffer cells, mouse livers were perfused in situ first with EGTA solution $\left(5.4 \mathrm{mM} \mathrm{KCl}, 0.44 \mathrm{mM} \mathrm{KH}_{2} \mathrm{PO}_{4}, 140 \mathrm{mM} \mathrm{NaCl}, 0.34 \mathrm{mM}\right.$ $\mathrm{Na}_{2} \mathrm{HPO}_{4}, 0.5 \mathrm{mM}$ EGTA, $25 \mathrm{mM}$ Tricine, $\mathrm{pH}$ 7.2), followed by the perfusion collagenase buffer (Worthington Biochemical Corporation, Lakewood, NJ, USA) and the digestion buffer $(0.009 \%$ collagenase type I in HBSS with $0.02 \%$ DNase I) at $37^{\circ} \mathrm{C}$ for $30 \mathrm{~min}$. The cell suspension was filtered through a $70 \mu \mathrm{m}$ cell strainer and centrifuged at $400 \mathrm{rpm}$ for 5 minutes at room temperature to remove hepatocytes. The supernatant was transferred to a new tube and centrifuged at $400 \mathrm{~g}$ for 10 minutes at $4{ }^{\circ} \mathrm{C}$, and then the pellet was resuspended in $6 \mathrm{ml}$ of $11.5 \%$ Optiprep (Sigma-Aldrich, St. Louis, MO, USA) and loaded carefully onto $6 \mathrm{ml}$ of $20 \%$ Optiprep and centrifuged at $1600 \mathrm{~g}$ for $17 \mathrm{~min}$ at $4{ }^{\circ} \mathrm{C}$. The cellular fraction in the interface between 11.5 and $20 \%$ Optiprep were gently collected. Finally, collected mouse and human liver tissues were dissociated by gentle MACS with Liver dissociation kit (Miltenyi Biotec, Bergisch Gladbach, Germany). Gathered liver tissues were washed with media (stable glutamine contained media; RPMI or low glucose DMEM), and were mixed with pre-warmed media and enzyme mixture. Then liver tissues were roughly chopped, and shook under gentle MACS dissociator according to the manufacturer's instructions.

BMDM generation. BMDMs were prepared. Collected bone marrow cells $(1.5 \times$ $10^{7}$ ) from the tibias and femurs of WT and Nox2 KO mice were seeded on $100 \mathrm{~mm}$ culture dish and incubated for 7 days in RPMI medium containing 30\% of L929 conditioned medium. BMDMs were gently harvested by scraping with a cell lifter. Collected BMDMs were resuspended in complete RPMI medium and counted for use in additional experiments.

Measurements of reactive oxygen species by palmitate. Infiltrated macrophages, resident Kupffer cells and RAW 264.7 cells (obtained from ATCC and free from mycoplasma) were stimulated with $200 \mu \mathrm{M}$ palmitate conjugated with BSA or only BSA for one hour, and surface immune-fluorescence antibody staining was performed quickly for identification of macrophages. The cells were incubated with ROS Detection Reagents; 6-chloromethyl-2', $7^{\prime}$-dichlorodihydrofluorescein diacetate, acetyl ester (CM- $\mathrm{H}_{2}$ DCF-DA from Invitrogen, Carlsbad, CA, USA) on $37^{\circ} \mathrm{C}$ for $15 \mathrm{~min}$, whereas incubated cells with PBS (Fluorescence Minus One; FMO) were used as negative control. This FMO control contained all the fluorochromes except for $\mathrm{CM}-\mathrm{H}_{2}$ DCF-DA that was being measure, and the fluorescence was 
slightly stronger than general unstained samples. Approximate fluorescence excitation is $492-495 \mathrm{~nm}$ and emission is $517-527 \mathrm{~nm}$, so oxidation of these probes can be detected by monitoring FITC fluorescence with FACS LSRII. Histogram of relative fluorescence intensities was used to compare ROS generation.

FACS analysis. Cells were labeled with fluorescence tagged antibodies; under the anti-mouse CD16/CD32 (mouse Fc blocker, Clone 2.4G2) (BD Pharmingen, San Jose, CA, USA) and the Live/dead fixable aqua dead cell stain kit for $405 \mathrm{~nm}$ excitation (Life Technologies, Carlsbad, CA, USA). Infiltrated macrophages $\left(\mathrm{CD} 11 \mathrm{~b}^{+}, \mathrm{F} 4 / 80^{+}\right)$and Kupffer cells $\left(\mathrm{CD} 11 \mathrm{~b}^{+}, \mathrm{F} 4 / 80^{\text {high }}\right)$ were described by using eFlour 450-conjugated anti-mouse CD45 (Clone 30-F11), FITC, PE, or PE/Cy7-F4/ 80 (Clone BM8) (eBioscience, San Diego, CA, USA), anti-mouse APC, APC-Cy7, or V500-CD11b (Clone M1/70) (BD Pharmingen, San Jose, CA, USA), and additional antibodies including anti-mouse FITC, PerCP-Cy5.5 or APC-Cy7-Ly-6C (Clone AL-21), and PE, PE-Cy7, or APC-Ly-6G (Clone 1A8) (BD bioscience, San Jose, CA, USA). Both cells were stained with anti-mouse APC-CD206 (MMR, Clone C068C2) (BioLegend, San Diego, CA, USA), PE or APC-conjugated TLR4/ MD-2 (Clone MTS510), PerCP-Cy5.5-CD14 (Clone rmC5-3), PerCP-Cy5.5-MHCII (I-A/I-E, Clone M5/114), PE-CD86 (Clone GL1), APC-CD11c (Clone HL3), FITC-CD1d (Clone 1B1) (BD Pharmingen, San Jose, CA, USA), anti-mouse FITCCD80 (Clone 16-10A1) (eBioscience, San Diego, CA, USA), and compared expression of these markers. Lymphocytes (CD4 T, CD8 T, NK, NKT cells), Granulocytes (monocytes, neutrophils etc.) and regulatory T cells $\left(\mathrm{CD} 4^{+}, \mathrm{CD} 25^{+}\right.$, $\mathrm{FoxP}^{+}$) were analyzed also through pseudo-color analysis plot. Stained cells read with FACS LSRII (BD Biosciences, San Jose, CA, USA), and the result was analyzed by FlowJo software (Flow Jo LLC, Ashland, OR, USA). Similarly, in human sample, infiltrated macrophages $\left(\mathrm{CD} 14^{\text {high }} \mathrm{CD} 68^{\text {low }}\right)$ and resident macrophages (CD14 $4^{\text {low }} \mathrm{CD} 68^{\text {high }}$ ) were distinguished by using anti-human BV421 or V450CD45 (Clone HI30) (BD Horizon, San Jose, CA, USA), anti-human PE or APCCy7-CD14 (Clone MФP9) (BD Pharmingen, San Jose, CA, USA), and anti-human FITC or PE-Cy7-CD68 (Clone eBioY1/82A) (eBioscience, San Diego, CA, USA) under the human Fc blocker (BD Pharmingen, San Jose, CA, USA). Relative TLR4 expression (APC-conjugated CD284, Clone HTA125) (eBioscience, San Diego, CA, USA) was observed with histogram plots.

Cell culture and palmitate treatment. RAW 264.7 cells (immortalized mouse monocyte cells) were obtained from American Type Culture Collection (ATTC, Manassas, VA, USA) and were cultured in Dulbecco's modified Eagle medium (WG, Gyeongsan, Korea) supplemented with $10 \%$ fetal bovine serum (WG, Gyeongsan, South Korea) and 1\% antibiotic-antimycotic (Thermo Fisher Scientific, Waltham, MA, USA) at $37^{\circ} \mathrm{C}$ with $5 \% \mathrm{CO}_{2}$. Used cells were confirmed to be free from mycoplasma. For preparation of palmitate treatment, stock solution of 100 $\mathrm{mM}$ palmitate (Sigma-Aldrich, St. Louis, MO, USA) was dissolved with $0.1 \mathrm{M}$ $\mathrm{NaOH}$ by heating at $70^{\circ} \mathrm{C}$ in a hot water bath without vortex. $5 \%$ BSA solution with fatty acid free (Sigma-Aldrich, St. Louis, MO, USA) was prepared with $\mathrm{H}_{2} \mathrm{O}$ and filtered through $0.22 \mu \mathrm{m}$ syringe filter. By mixing two solutions, $5 \mathrm{mM}$ palmitate-BSA was conjugated in a $55^{\circ} \mathrm{C}$ water bath for $10-15$ minutes, and cooling down before use. In some cases, $10 \mu \mathrm{M}$ sulfosuccinimidyl oleate (SSO) (Cayman, Ann Arbor, MI, USA) or $80 \mu \mathrm{M}$ dynasore (Sigma-Aldrich, St. Louis, MO, USA) was pre-treated for $10 \mathrm{~min}$ before palmitate treatment.

Immunofluorescence staining. For immunofluorescence staining, cells were plated in adhesive slides by using Cytospin centrifugation according to the manufacturer's instructions (Thermo Fisher Scientific, Waltham, MA, USA), and fixed with cold methanol $(99 \%)$ for $10 \mathrm{~min}$ at $4{ }^{\circ} \mathrm{C}$. After fixation, $0.1 \%$ Tween 20 (Sigma-Aldrich, St. Louis, MO, USA) was applied for permeabilization. Cells were incubated with primary antibodies of TLR4 (\#293072) (Santa Cruz Biotechnology, CA, USA) and NOX2 (\#ab31902) (Abcam, Cambridge, UK) for overnight at $4{ }^{\circ} \mathrm{C}$ and they were incubating with Alexa Fluor 488-conjugated anti-mouse IgG (\#ab15109) or 594-conjugated anti-rabbit IgG (\#ab150064) (Abcam, Cambridge, UK) for one hour at room temperature and mounted VECTASHIELD medium with DAPI (Vector laboratories, Burlingame, CA, USA) following the manufacturer's instructions. Fluorescence images were acquired using an Olympus BX51 microscope equipped with a CCD camera (Olympus, Tokyo, Japan) and computerassisted image analysis with DP2-BSW.

Ligand-dependent dimerization of hTLR4-MD2 complex. Human TLR4 (amino acids 27-631) and protein A tagged MD2 at C-termini (amino acids 19-160) were co-expressed in High Five insect cells (Invitrogen, Carlsbad, CA, USA). The hTLR4-MD2-protein A complex was purified by IgG Sepharose affinity chromatography (GE Healthcare Life Sciences, Chicago, IL, USA) and the protein A tag was cleaved off with thrombin digestion on resin. The hTLR4-MD2 complex was subjected to gel filtration chromatography with Superdex 200 column (GE Healthcare Life Sciences, Chicago, IL, USA). hTLR4/MD2 complex $(6.85 \mu \mathrm{M})$ was incubated with $68.5 \mu \mathrm{M}$ of various ligands (LPS Ra, C16-BODIPY, Palmitate-BSA, BSA) for $3 \mathrm{~h}$ at $37^{\circ} \mathrm{C}$. The ligands were sonicated for $10 \mathrm{~min}$ prior to the reaction. The mixture of hTLR4-MD2 complex and each ligand was applied to Superdex 200 for gel filtration chromatography. The elution fractions were analyzed with SDS-PAGE followed by silver staining. Elution fractions of hTLR4-MD2 complex with C16-BODIPY (Invitrogen, Carlsbad, CA, USA) were further analyzed by native PAGE in which the bands corresponding C16-BODIPY bound TLR4-MD2 were visualized with subsequent Coomassie (Thermo Fisher Scientific, Waltham, MA, USA) staining and illumination at $488 \mathrm{~nm}$.

Binding assay between palmitate and TLR4/MD2 protein. Purified protein (mouse and human TLR4, MD2, TLR4-MD2 complex, CD14) was incubated with $200 \mu \mathrm{M}$ fluorescence tagged palmitic acid (BODIPY) in room temperature for 30 min. The samples were loaded on gradient Native PAGE gel (4-15\%) (Koma Biotech, Seoul, South Korea) at $4{ }^{\circ} \mathrm{C}$.

TUNEL staining. Apoptotic cells were detected by In situ Cell Death Detection Kit (Roche, IN, USA) according to the manufacturer's instructions. In brief, deparaffinized liver sections were pre-treated with proteinase K (Sigma-Aldrich, St. Louis, MO, USA). The slide was incubated with TdT reaction solution in humidified chamber for $2 \mathrm{~h}$ and stop reaction. Positive cells were stained with FITCAvidin D, and counter-staining was performed with DAPI. The number of positive cells was counted per equivalent area.

Annexin $\mathbf{V}$ binding assay. Early stage of apoptosis was analyzed by Annexin $\mathrm{V}$ and 7-Amino-Actinomycin (7-AAD) staining (BD Pharmingen, San Jose, CA, USA). After palmitate treatment for $1 \mathrm{~h}$, macrophages and Kupffer cells were incubated with FITC-Annexin V and 7-AAD for $15 \mathrm{~min}$ in Annexin V binding buffer (BD Pharmingen, San Jose, CA, USA). Early apoptosis was represented as Annexin $\mathrm{V}$ positive and $7-\mathrm{AAD}$ negative $\left(\mathrm{Annexin} \mathrm{V}^{+} 7-\mathrm{AAD}^{-}\right)$.

Human liver samples. HCC tissue samples were obtained from the archives of the Department of Surgery, Chungnam National University Hospital (Daejeon, South Korea). HCC blood samples were obtained from the archives of the Division of Gastroenterology, Department of Internal Medicine, Chungnam National University Hospital. HCC tissues were subjected to fluorescence-activated cell sorting for CD68 and CD14 analyses. Peripheral blood mononuclear cells (PBMCs) from blood samples of healthy donors were isolated and analyzed. Purified CD14+ (Magnisort; affymetrix, Santa Clara, CA, USA) PBMCs were applied to ROS measurement after palmitate or palmitate treatment with dynasore pre-incubated or not. Authorization for the use of these tissues for research purposes and ethical approval were obtained from the Institutional Review Board of Chungnam National University Hospital (IRB number: 2016-03-02-003). Informed consents, which were approved by Institutional Review Board of Chungnam National University Hospital, were received from the entire patients who had provided the tissue or blood. Non-tumor lesions of liver tissue samples from a patient with fatty liver and eight patients with HCC $(n=6)$ or metastasis $(n=2)$ from colorectal cancer of stage IV were used in this research. All of these patients had undergone tumor resection operation between 2016 and 2017.

Gene Expression Omnibus data set. In order to analyze the expression level of genes in NASH liver, gene expression data were obtained from the National Center for Biotechnology Information (NCBI) Gene Expression Omnibus (GEO) database (accession code GSE63067) and previously published microarray data. The GSE63067 data set includes two human samples of steatosis and nine human samples for non-alcoholic steatohepatitis collected at the Swedish University of Agricultural Sciences, and it was used to define the molecular characteristics and to define non-alcoholic steatohepatitis early markers from Steatosis ${ }^{52}$. Nine NASH samples in the cohort were taken into consideration for analyses as compared with normal control.

Statistical analysis. All data are presented as the mean \pm s.e.m. The statistical differences between groups were determined by the Student's t-test or one-way analysis of variance for multiple comparisons using GraphPad Prism 5 (GraphPad Software, La Jolla, CA, USA). Differences were considered statistically significant at $P<0.05$.

Data availability. All relevant data that support the findings of this study are available from the corresponding author upon request.

Received: 9 May 2017 Accepted: 21 November 2017

Published online: 21 December 2017

\section{References}

1. Talukdar, S. et al. Neutrophils mediate insulin resistance in mice fed a high-fat diet through secreted elastase. Nat. Med. 18, 1407-1412 (2012).

2. Osborn, O. \& Olefsky, J. M. The cellular and signaling networks linking the immune system and metabolism in disease. Nat. Med. 18, 363-374 (2012).

3. Olefsky, J. M. \& Glass, C. K. Macrophages, inflammation, and insulin resistance. Annu. Rev. Physiol. 72, 219-246 (2010). 
4. Krenkel, O. \& Tacke, F. Liver macrophages in tissue homeostasis and disease. Nat. Rev. Immunol. 17, 306-321 (2017).

5. Nguyen, M. T. et al. A subpopulation of macrophages infiltrates hypertrophic adipose tissue and is activated by free fatty acids via Toll-like receptors 2 and 4 and JNK-dependent pathways. J. Biol. Chem. 282, 35279-35292 (2007).

6. Guichard, C., Moreau, R., Pessayre, D., Epperson, T. K. \& Krause, K. H. NOX family NADPH oxidases in liver and in pancreatic islets: a role in the metabolic syndrome and diabetes? Biochem. Soc. Trans. 36, 920-929 (2008).

7. Santos, C. X., Tanaka, L. Y., Wosniak, J. \& Laurindo, F. R. Mechanisms and implications of reactive oxygen species generation during the unfolded protein response: roles of endoplasmic reticulum oxidoreductases, mitochondrial electron transport, and NADPH oxidase. Antioxid. Redox Signal. 11, 2409-2427 (2009).

8. Bedard, K. \& Krause, K. H. The NOX family of ROS-generating NADPH oxidases: physiology and pathophysiology. Physiol. Rev. 87, 245-313 (2007).

9. Meng, T. et al. Propofol reduces lipopolysaccharide-induced, NADPH oxidase (NOX 2) mediated TNF- alpha and IL-6 production in macrophages. Clin. Dev. Immunol. 2013, 325481 (2013).

10. Thakur, V., Pritchard, M. T., McMullen, M. R., Wang, Q. \& Nagy, L. E. Chronic ethanol feeding increases activation of NADPH oxidase by lipopolysaccharide in rat Kupffer cells: role of increased reactive oxygen in LPS-stimulated ERK1/2 activation and TNF-alpha production. J. Leukoc. Biol. 79, 1348-1356 (2006).

11. Bae, Y. S. et al. Macrophages generate reactive oxygen species in response to minimally oxidized low-density lipoprotein: toll-like receptor 4- and spleen tyrosine kinase-dependent activation of NADPH oxidase 2. Circ. Res. 104, 210-218 (2009).

12. Kuchler, L. et al. SYNCRIP-Dependent Nox2 mRNA Destabilization Impairs ROS Formation in M2-Polarized Macrophages. Antioxid. Redox. Signal. 21, 2483-2497 (2014).

13. Ryu, J. K. et al. Reconstruction of LPS Transfer Cascade Reveals Structural Determinants within LBP, CD14, and TLR4-MD2 for Efficient LPS Recognition and Transfer. Immunity 46, 38-50 (2017).

14. Gomez, R., Villalvilla, A., Largo, R., Gualillo, O. \& Herrero-Beaumont, G. TLR4 signalling in osteoarthritis--finding targets for candidate DMOADs. Nat. Rev. Rheumatol. 11, 159-170 (2015).

15. Husebye, H. et al. Endocytic pathways regulate Toll-like receptor 4 signaling and link innate and adaptive immunity. EMBO J. 25, 683-692 (2006).

16. Canton, J. \& Grinstein, S. Priming and activation of NADPH oxidases in plants and animals. Trends Immunol. 35, 405-407 (2014).

17. Moon, J. S. et al. NOX4-dependent fatty acid oxidation promotes NLRP3 inflammasome activation in macrophages. Nat. Med. 22, 1002-1012 (2016).

18. Ngkelo, A., Meja, K., Yeadon, M., Adcock, I. \& Kirkham, P. A. LPS induced inflammatory responses in human peripheral blood mononuclear cells is mediated through NOX4 and Gialpha dependent PI-3kinase signalling. J. Inflamm. 9, 1 (2012).

19. Holt, M. P., Cheng, L. \& Ju, C. Identification and characterization of infiltrating macrophages in acetaminophen-induced liver injury. J. Leukoc. Biol. 84, 1410-1421 (2008).

20. Kim, H. M. et al. Crystal structure of the TLR4-MD-2 complex with bound endotoxin antagonist Eritoran. Cell 130, 906-917 (2007).

21. Thumser, A. E. \& Storch, J. Characterization of a BODIPY-labeled fluorescent fatty acid analogue. Binding to fatty acid-binding proteins, intracellular localization, and metabolism. Mol. Cell. Biochem. 299, 67-73 (2007).

22. Seo, W. et al. Exosome-mediated activation of toll-like receptor 3 in stellate cells stimulates interleukin-17 production by gammadelta $\mathrm{T}$ cells in liver fibrosis. Hepatology 64, 616-631 (2016).

23. Pepping, J. K., Freeman, L. R., Gupta, S., Keller, J. N. \& Bruce-Keller, A. J. NOX2 deficiency attenuates markers of adiposopathy and brain injury induced by high-fat diet. Am. J. Physiol. Endocrinol. Metab. 304, E392-E404 (2013).

24. Ronis, M. J. et al. Female mice lacking p47phox have altered adipose tissue gene expression and are protected against high fat-induced obesity. Physiol. Genomics 45, 351-366 (2013).

25. Fukuda, M. et al. Ezetimibe ameliorates cardiovascular complications and hepatic steatosis in obese and type 2 diabetic $\mathrm{db} / \mathrm{db}$ mice. J. Pharmacol. Exp. Ther. 335, 70-75 (2010).

26. Costford, S. R. et al. Mice lacking NOX2 are hyperphagic and store fat preferentially in the liver. Am. J. Physiol. Endocrinol. Metab. 306, E1341-E1353 (2014).

27. Motoyama, K. et al. Isolation stress for 30 days alters hepatic gene expression profiles, especially with reference to lipid metabolism in mice. Physiol. Genomics 37, 79-87 (2009).

28. Sakakibara, H. et al. Social isolation stress induces hepatic hypertrophy in C57BL/6J mice. J. Toxicol. Sci. 37, 1071-1076 (2012).

29. Urao, N. et al. Role of nox2-based NADPH oxidase in bone marrow and progenitor cell function involved in neovascularization induced by hindlimb ischemia. Circ. Res. 103, 212-220 (2008).

30. Stewart, C. R. et al. CD36 ligands promote sterile inflammation through assembly of a Toll-like receptor 4 and 6 heterodimer. Nat. Immunol. 11 155-161 (2010).
31. Wang, Y. C. et al. Toll-like receptor $2 / 4$ heterodimer mediates inflammatory injury in intracerebral hemorrhage. Ann. Neurol. 75, 876-889 (2014).

32. Zanoni, I. et al. CD14 controls the LPS-induced endocytosis of Toll-like receptor 4. Cell 147, 868-880 (2011).

33. Tan, Y., Zanoni, I., Cullen, T. W., Goodman, A. L. \& Kagan, J. C. Mechanisms of Toll-like Receptor 4 Endocytosis Reveal a Common Immune-Evasion Strategy Used by Pathogenic and Commensal Bacteria. Immunity 43, 909-922 (2015).

34. Erridge, C. \& Samani, N. J. Saturated fatty acids do not directly stimulate Tolllike receptor signaling. Arterioscler. Thromb. Vasc. Biol. 29, 1944-1949 (2009).

35. Lamb, F. S. et al. Endotoxin priming of neutrophils requires endocytosis and NADPH oxidase-dependent endosomal reactive oxygen species. J. Biol. Chem. 287, 12395-12404 (2012).

36. Singleton, P. A. et al. Dynamin 2 and c-Abl are novel regulators of hyperoxiamediated NADPH oxidase activation and reactive oxygen species production in caveolin-enriched microdomains of the endothelium. J. Biol. Chem. 284, 34964-34975 (2009).

37. Wang, Y. et al. Saturated palmitic acid induces myocardial inflammatory injuries through direct binding to TLR4 accessory protein MD2. Nat. Commun. 8, 13997 (2017).

38. Jin, S., Zhou, F., Katirai, F. \& Li, P. L. Lipid raft redox signaling: molecular mechanisms in health and disease. Antioxid. Redox Signal. 15, 1043-1083 (2011).

39. Szabo, G., Dolganiuc, A., Dai, Q. \& Pruett, S. B. TLR4, ethanol, and lipid rafts: a new mechanism of ethanol action with implications for other receptormediated effects. J. Immunol. 178, 1243-1249 (2007).

40. Plociennikowska, A., Hromada-Judycka, A., Borzecka, K. \& Kwiatkowska, K. Co-operation of TLR4 and raft proteins in LPS-induced pro-inflammatory signaling. Cell. Mol. Life. Sci. 72, 557-581 (2015).

41. Snodgrass, R. G., Huang, S., Choi, I. W., Rutledge, J. C. \& Hwang, D. H. Inflammasome-mediated secretion of IL-1beta in human monocytes through TLR2 activation; modulation by dietary fatty acids. J. Immunol. 191, 4337-4347 (2013).

42. Reynolds, C. M. et al. Dietary saturated fatty acids prime the NLRP3 inflammasome via TLR4 in dendritic cells-implications for diet-induced insulin resistance. Mol. Nutr. Food Res. 56, 1212-1222 (2012).

43. Lackey, D. E. \& Olefsky, J. M. Regulation of metabolism by the innate immune system. Nat. Rev. Endocrinol. 12, 15-28 (2016).

44. Li, Y. et al. Inhibition of mitochondrial fission and NOX2 expression prevent NLRP3 inflammasome activation in the endothelium: the role of corosolic acid action in the amelioration of endothelial dysfunction. Antioxid. Redox. Signal. 24, 893-908 (2016).

45. Pal, R., Basu Thakur, P., Li, S., Minard, C. \& Rodney, G. G. Real-time imaging of NADPH oxidase activity in living cells using a novel fluorescent protein reporter. PLoS ONE 8, e63989 (2013).

46. Lew, W. Y. et al. Myocardial fibrosis induced by exposure to subclinical lipopolysaccharide is associated with decreased miR-29c and enhanced NOX2 expression in mice. PLoS ONE 9, e107556 (2014).

47. Gandhirajan, R. K. et al. Blockade of NOX2 and STIM1 signaling limits lipopolysaccharide-induced vascular inflammation. J. Clin. Invest. 123, 887-902 (2013).

48. Souto Padron de Figueiredo, A. et al. Nox2 mediates skeletal muscle insulin resistance induced by a high fat diet. J. Biol. Chem. 290, 13427-13439 (2015).

49. Judkins, C. P. et al. Direct evidence of a role for Nox2 in superoxide production, reduced nitric oxide bioavailability, and early atherosclerotic plaque formation in $\mathrm{ApoE}^{-/-}$mice. Am. J. Physiol. Heart Circ. Physiol. 298, H24-H32 (2010).

50. Yuan, $\mathrm{H}$. et al. NADPH oxidase 2-derived reactive oxygen species mediate FFAs-induced dysfunction and apoptosis of beta-cells via JNK, p38 MAPK and p53 pathways. PLoS ONE 5, e15726 (2010).

51. Yang, C. S. et al. NADPH oxidase 2 interaction with TLR2 is required for efficient innate immune responses to mycobacteria via cathelicidin expression. J. Immunol. 182, 3696-3705 (2009).

52. Frades, I. et al. Integrative genomic signatures of hepatocellular carcinoma derived from nonalcoholic Fatty liver disease. PLoS ONE 10, e0124544 (2015)

\section{Acknowledgements}

This work was supported by the National Research Foundation of Korea (NRF) grant funded by the Korean government (MSIP) (NFR-2015R1A2A1A10055551), the Korea Mouse Phenotyping Project (NRF-2014 M3A9D5A01073556) of the Ministry of Science, ICT and Future Planning through the National Research Foundation, and the Intelligent Synthetic Biology Center of Global Frontier Project funded by the Ministry of Science, ICT \& Future Planning (2011-0031955), Republic of Korea.

\section{Author contributions}

S.Y.K., J.-M.J., W.S., and W.-I.J. contributed to design and performance of experiments and analyses of data. M.-H.K., W.-M.C. J.-H.L., and Y.-R.S. contributed to isolation of mouse macrophages and flow cytometry analyses. W.Y. contributed to analyses of GEO database. S.Y.K., H.-S.Y. and Y.-S.L. contributed to isolation of human macrophages and 
PBMCs from patients and to flow cytometry analyses. H.S.E., B.S.L., and K.C. contributed to collection of human liver samples and analyses of data. S.-J.K. and H.M.K performed size exclusive chromatography and analyses of data. S.C.K., B.G., G.K., H.M.K., and W.-I.J. wrote the manuscript.

\section{Additional information}

Supplementary Information accompanies this paper at https://doi.org/10.1038/s41467017-02325-2.

Competing financial interests: The authors declare no competing financial interests

Reprints and permission information is available online at http://npg.nature.com/ reprintsandpermissions/

Publisher's note: Springer Nature remains neutral with regard to jurisdictional claims in published maps and institutional affiliations. (c) (i) Open Access This article is licensed under a Creative Commons Attribution 4.0 International License, which permits use, sharing, adaptation, distribution and reproduction in any medium or format, as long as you give appropriate credit to the original author(s) and the source, provide a link to the Creative Commons license, and indicate if changes were made. The images or other third party material in this article are included in the article's Creative Commons license, unless indicated otherwise in a credit line to the material. If material is not included in the article's Creative Commons license and your intended use is not permitted by statutory regulation or exceeds the permitted use, you will need to obtain permission directly from the copyright holder. To view a copy of this license, visit http://creativecommons.org/ licenses/by/4.0/.

(C) The Author(s) 2017 